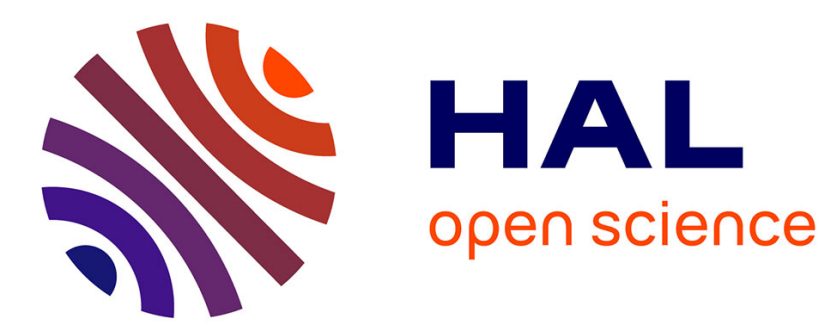

\title{
Inverse rheometry and basal properties inference for pseudoplastic geophysical flows
}

\author{
Nathan Martin, Jerome Monnier
}

\section{To cite this version:}

Nathan Martin, Jerome Monnier. Inverse rheometry and basal properties inference for pseudoplastic geophysical flows. European Journal of Mechanics - B/Fluids, 2015, 50, pp.110 - 126. 10.1016/j.euromechflu.2014.11.011 . hal-01095602

\section{HAL Id: hal-01095602 \\ https://hal.science/hal-01095602}

Submitted on 15 Dec 2014

HAL is a multi-disciplinary open access archive for the deposit and dissemination of scientific research documents, whether they are published or not. The documents may come from teaching and research institutions in France or abroad, or from public or private research centers.
L'archive ouverte pluridisciplinaire HAL, est destinée au dépôt et à la diffusion de documents scientifiques de niveau recherche, publiés ou non, émanant des établissements d'enseignement et de recherche français ou étrangers, des laboratoires publics ou privés. 


\title{
Inverse rheometry and basal properties inference for pseudoplastic geophysical flows
}

\author{
N. Martin ${ }^{\mathrm{a}}$ J. Monnier ${ }^{\mathrm{b}}$ \\ ${ }^{a}$ California Institute of Technology's Jet Propulsion Laboratory, 4800 Oak Grove Drive, Pasadena, CA 91109, United States \\ ${ }^{b}$ Mathematics Institute of Toulouse - National Institute for Applied Sciences, 135 Avenue de Rangueil, 31077, Toulouse Cedex 4, France
}

\begin{abstract}
The present work addresses the question of performing inverse rheometry and basal properties inference for pseudoplastic gravity-driven free-surface flows at low Reynolds' number. The modeling of these flows involves several parameters, such as the rheological ones or the state of the basal boundary (modeling an interface between the base and the fluid). The issues of inverse rheometry are addressed in a general laboratory flow context using surface velocity data. The inverse characterization of the basal boundary is proposed in a geophysical flow context where the parameters involved in the empirical effective sliding law are particularly difficult to estimate. Using an accurate direct and inverse model based on the adjoint method combined with an original efficient solver, sensitivity analyses and parameter identification are performed for a wide range of flow regimes, defined by the degree of slip and the non-linearity of the viscous sliding law considered at the bottom.

The first result is the numerical assessment of the passive aspect of the viscosity singularity inherent to a powerlaw pseudoplastic (shear-thinning) description in terms of surface velocities. From this result, identification of the two parameters of the constitutive law, namely the power-law exponent and the consistency, are performed. These numerical experiments provide, on the one hand, a very robust identification of the power-law exponent, even for very noisy surface velocity observations and on the other hand, a strong equifinality problem on the identification of the consistency. This parameter has a minor influence on the flow, in terms of surface velocities. Typically for temperature-dependent geophysical fluids, a law describing a priori its spatial variability is then sufficient (e.g. based on a temperature vertical profile).

This study then focuses on the basal properties interacting with the fluid rheology. An accurate joint identification of the scalar valued triple $(n, m ; \beta)$ (respectively the rheological exponent, the non linear friction exponent and the friction coefficient) is achieved for any degree of slip, allowing to completely infer the flow regime. Next, in a geophysical flow context, identifications of a spatially varying friction coefficient are performed for various perturbed bedrock topography. The (2D-vertical) results demonstrates a severely ill-posed problem that allows to compute a given set of surface velocity data with different topography/friction pairs.
\end{abstract}

\section{Introduction}

Power-law fluids represent a wide category of materials in the range of shear rates to which the coefficients were fitted. Pseudoplastic fluids designate fluids presenting a shear-thinning behavior, modeled by a powerlaw constitutive law (e.g. polymer solutions, ice, blood etc) which expresses the relationship between the deviatoric stress tensor $\underline{\boldsymbol{S}}$ and the strain-rate tensor $\underline{\boldsymbol{D}}$ as (see also Section 2.1):

$$
\underline{\boldsymbol{S}}=2 \eta_{0}\|\underline{\boldsymbol{D}}\|_{F}^{\frac{1-n}{n}} \underline{\boldsymbol{D}}
$$

The power-law type description leads to focus on the two undetermined parameters of such a law which are the exponent of the law $n$ (scalar value) and the rate factor or consistency $\eta_{0}$ (possibly spatially distributed) which are generally hard to estimate in a real context and difficult to measure experimentally (see e.g. [1]). In a geophysical context, these fluids involve gravitydriven mass movements and are generally treated as fluids flowing down a slope (see e.g. [2]). They show a complex and non uniform rheology and have a strong dependency on their basal properties, possibly in relation with their rheology. The basal properties mainly involve the modeling of a basal slip through a (possibly non-linear) viscous empirical effective sliding law. The 
sliding law is itself a model. It expresses the relationship between the basal shear $\sigma_{\text {nt }}$ and the basal velocity $\mathbf{u} \cdot \mathbf{t}$ as (see also Section 2.1):

$$
\left|\sigma_{\mathbf{n t}}\right|^{m-1} \sigma_{\mathbf{n t}}=\beta \mathbf{u} \cdot \mathbf{t}
$$

It includes a friction parameter $\beta$ which is hard to directly estimate and an exponent $m$ describing the nonlinear response of the subglacial material on which the sliding occurs. It is in this context that the possibility of inferring these quantities (and consequently the model they describe) through inverse modeling becomes essential.

In the case of scalar valued parameters, the identification of rheological components formulated as an inverse problem (thus using a direct differentiation of the problem) has been treated in an industrial context (particularly metal forming), adapted to particular experimental setups only. Following this type of approach, identification of rheological parameters based on cross-section velocity measurements can be found in $[3,4]$. A similar approach using measurement of a pressure drop is proposed by [5]. In [6], attention is also payed to the identification of a scalar friction parameter.

The present study aims to perform inverse rheometry in a more general context, hence applicable to broader experimental setups. In addition, the present study focus on geophysical flows whose characteristics are: uniqueness of a given situation (compared to reproducible laboratory experiments), velocity observation generally limited to the surface and a possible strong influence of an unknown and unmeasurable basal slip modeling an heterogeneous basal interface non linearly interacting with the bed and the flow. We focus hereafter on a pseudoplastic representative geophysical examples which is ice flows. In ice flows modeling, the power-law model firstly presented in [7] is well admitted; while its temperature and shear rate dependency is still a matter of debate (see e.g. [8], [9]). The coupling with thermal physics also occurs in the definition of the consistency.

For instance, the present equations are also suited for modeling lava flows. In this case, the question of the power-law index value is still under debate and has been widely discussed but the literature generally agrees on a pseudoplastic (shear-thinning) behavior of the magma with possible dependency of this exponent on temperature and/or crystal concentration (see e.g. [10]).
The present studies are mainly led in this geophysical fluid flow context, but the method and the results, when mentioned, can be extended to general experimentally controlled flows in laboratory experiments. Also, the model and methods developed here are also valid for dilatant (shear-thickening) fluids but has not been numerically explored.

If the fluid flow present basal slip, the latter becomes a major component of its modeling. Thus, investigations regarding the physical components and relevant parameterizations introduced in the (empirical effective) friction law are of primal importance.

In the case of ice and lava, different laws are used to evaluate this parameter but a common description is to consider an Arrhenius-type law including appropriate physical considerations, see e.g. [8] for ice and [11] for magma. In glaciology, the basal friction law models the subglacial water pressure, underlying non linear till, surface roughness, geothermal heat flux etc, see eg [8]. The identification of the basal friction coefficient and the consistency in glaciers models using a variational approach became quite common recently but considering the ice viscosity to be independent of the velocity in the "adjoint model" derivation, hence simplifying greatly the inverse model but leading to the computation of an incorrect gradient. The continuous adjoint model in the glaciological context, with nonlinear basal slip, can be found in [12]. In other respect, the question of basal properties characterization through surface velocity observations using the present variational approach is studied in [13].

In a computational point of view, the present study is based on a second-order forward and adjoint solution for the power-law Stokes problem. The forward problem, written as a four-field saddle point formulation of the power-law Stokes problem, is discretized with three-field finite elements and solved using a splitting technique and an augmented Lagrangian approach (providing large cpu-time saving, see [14]). The adjoint problem is solved using source-to-source automatic differentiation of the augmented Lagrangian algorithm. The newly obtained adjoint solver provides a second order accuracy gradient, efficiently computed and with low memory needs. Ratios of four (4) in cpu-time and memory consumption are assessed, compared to the differentiation of a classical fixed-point based solver. We refer to [13] for the technical method to assess the gradient accuracy. 
In any case, what can be obtained from an inverse approach is strongly related with the type and quality of available data. This study focuses on the use of surface horizontal velocity data (generally the most dense and accurate data in the present geophysical context, see e.g. [15]) to constrain the rheology and the basal properties of this type of flow depending on the flow regime. All the numerical experiments are designed according to the bounds in terms of basal variability transmission to the surface assessed in [13]. The experiments are carried out on steady state geometries of the free-surface gravity-driven flows.

As a preliminary issue, this study numerically address the role of the well-known viscosity singularity at the surface (i.e. at vanishing shear-rate) in the power-law shear-thinning constitutive law, in an inverse context, relying on surface velocity observations. As a matter of fact, this singularity might lead to an ill-posed direct problem if a free-surface is considered as pointed out in [16]. For different sliding regimes, sensitivity analysis demonstrate that the refinement of the mesh close to the surface, while leading to the appearance of a stiff (very high viscosity) layer, does not affect the solution and that the viscosity singularity remains passive in the model confirming numerically the analysis done in [17].

From this result, numerical experiments of virtual rheometry are carried out. Identifications of the scalar power-law exponent first, and then sensitivity analysis and identification of the spatially distributed consistency are performed. The identification of the power-law exponent proves to be extremely robust (providing a highly accurate identification for a $50 \%$ noise on the data), regardless of the flow regime. Conversely, the identification of a temperature-dependent consistency is a severely ill-posed problem, even with very good prior physical intuitions introduced in the initial guess and the regularization term, leading to a strong equifinality issue. We conclude that the consistency has a negligible role in the model in the bulk and that a trustworthy modeling a priori of the temperature-dependency is sufficient.

Based on this result, we then perform identifications of the scalar-valued triple $(n, m ; \beta)$ (respectively the power-law exponent, the friction law exponent and the friction coefficient) for all situations of sliding (from strong friction to high sliding). The results are fully conclusive allowing to accurately infer these three scalar quantities and therefore the regime of the flow, based only on surface velocity observations.

The final step is to identify (typically using the scalar identification as a very good initial guess) a spatially varying friction coefficient $\beta$. The numerical experiment is designed in order to explore the typical regimes of sliding of an ice-stream flow (from medium friction to very rapid sliding), with relevent length scales for the basal variability chosen following [13]. Based on a reference simulation, representing a "true state", simulations on randomly perturbed bedrock topographies are performed. The main result is the fact that the inferred friction coefficient completely depends on the topography in order to produce a given set of surface velocity data. This final result point a strong equifinality problem for the topography/friction pair and raises a question on how it affects the predictive capabilities of ice flow models since the most poorly observed data is the bedrock topography.

\section{Forward Model}

In this section we present the key aspects of the fluid model considered in the present study and its numerical approximation.

\subsection{Continuous Model}

The flow is assumed to be $2 \mathrm{D}$ vertical (in $(x, z)$ coordinates); it is modelled as an incompressible viscous fluid in a time moving domain $\Omega_{t}$ open, bounded and connected in $R^{2}$ with boundary $\partial \Omega$ (assumed to be Lipschitz). The momentum balance is described using the incompressible Navier-Stokes equation with low Reynolds approximation, thus by the so-called Stokes equation. The flow is driven by the gravity source term. The forward model equations are:

$$
\begin{aligned}
-\operatorname{div}(\underline{\sigma}) & =\rho \mathbf{g} \text { in } \Omega_{t} \\
\operatorname{div}(\mathbf{u}) & =0 \text { in } \Omega_{t}
\end{aligned}
$$

where $\mathbf{u}=\left(u_{x}(x, z, t), u_{z}(x, z, t)\right) \forall(x, z) \in \Omega_{t}, t \in$ $[0, T]$ denotes the velocity, $\boldsymbol{\sigma}$ the Cauchy stress tensor, $\rho$ the fluid density, $\mathbf{g}$ the gravity and $\Omega_{t}$ is the domain at time $t$. The stress tensor $\underline{\sigma}$ is decomposed as:

$$
\sigma=-p \underline{I d}+\underline{\boldsymbol{S}}
$$

where $\underline{S}$ is the deviatoric (or extra) stress tensor, $p$ the pressure and $\underline{I} \boldsymbol{d}$ the unit tensor. The non Newtonian viscous behavior is described by a viscoplastic constitutive law that relates the stress tensor to the strain-rate tensor. A power-law model, first proposed by Ostwald in 1925 


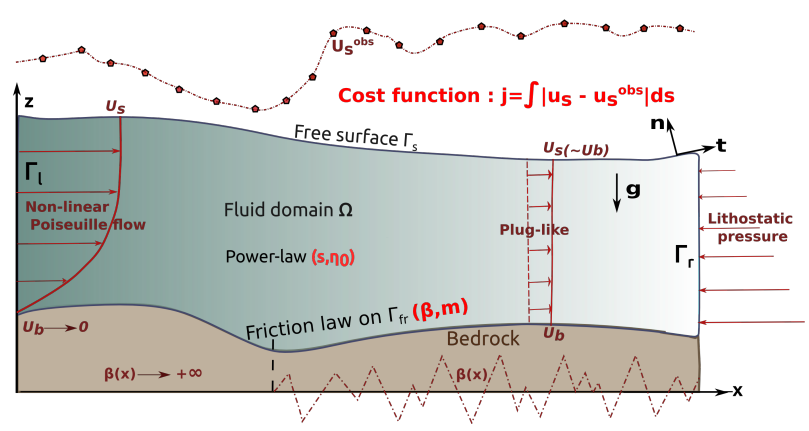

Figure 1: Typical geometry of the free-surface geophysical flow considered. Notations of the geometry and the boundary conditions. Schematic representation of the inverse problem where $u_{s}$ and $u_{b}$ designates surface velocity and basal velocity respectively and $u_{s}^{o b s}$ designates isolated surface velocity measurements. The control variables are represented in bold red as well as the typical cost function and are $\beta, m, n$ and $\eta_{0}$ (see end of Section 3.2).

(see e.g. [18]), is considered hereafter. It follows that the deviatoric tensor $\underline{S}$ is written as:

$$
\underline{\boldsymbol{S}}=2 \eta(\mathbf{u}) \underline{\boldsymbol{D}} \text { with } \eta(\mathbf{u})=\eta_{0}\|\underline{\boldsymbol{D}}\|_{F}^{\frac{1-n}{n}}
$$

where $\eta$ is the apparent viscosity, $\underline{\boldsymbol{D}}=\frac{1}{2}\left(\nabla \mathbf{u}+\nabla \mathbf{u}^{T}\right)$ the strain-rate tensor, $n$ the power-law index and the consistency $\eta_{0}$ (also called rate or creep factor in glaciology) is a dimensional constant which can depend on the state of the considered fluid (typically the temperature). The Frobenius matrix norm $\|\cdot\|_{F}$ usually called the shearrate is defined by:

$$
\|\underline{D}\|_{F}=\sqrt{\frac{1}{2} \operatorname{tr}\left(\underline{D} \underline{D}^{T}\right)}=\dot{\gamma}
$$

A typical domain is represented in Figure 1. The surface $\Gamma_{s}$, considered to be the graph of a function $h(x, t)$, is a free surface moving in time. The boundary $\partial \Omega$ is divided as follows: $\Gamma_{s}$ is the moving surface (free surface), $\Gamma_{b}$ is the bottom surface, $\Gamma_{l}$ and $\Gamma_{r}$ are lateral open boundaries.

The free surface dynamic is described by the following $1 \mathrm{D}$ transport equation:

$$
\partial_{t} h+u_{x} \partial_{x} h=a+u_{z} \text { on } \Gamma_{s} \times[0, T]
$$

with $a$ modelling a mass balance source term.

\section{Boundary conditions.}

We introduce $(\mathbf{t}, \mathbf{n})$, the normal-tangent pair of unit vectors such that:

$$
\sigma=(\sigma \cdot \mathbf{n}) \mathbf{n}+(\sigma \cdot \mathbf{t}) \mathbf{t}
$$

with:

$$
\sigma \cdot \mathbf{n}=\sigma_{n n} \mathbf{n}+\sigma_{n t} \mathbf{t} \quad, \quad \sigma \cdot \mathbf{t}=\sigma_{t n} \mathbf{n}+\sigma_{t t} \mathbf{t}
$$

On the top surface $\Gamma_{s}$ we consider a traction-free boundary condition while given stress or Dirichlet conditions can be prescribed on the lateral boundaries $\Gamma_{l}$ and $\Gamma_{r}$. The boundary $\Gamma_{b}$ is splitted into an homogeneous Dirichlet area $\Gamma_{a d}$ and a friction (or sliding) area $\Gamma_{f r}$. The friction/sliding law on $\Gamma_{f r}$ is a power-law type law (also known as Weertman law, see e.g. [8]) given by:

$$
\left|\sigma_{\mathbf{n t}}\right|^{m-1} \sigma_{\mathbf{n t}}=\beta \mathbf{u} \cdot \mathbf{t} \text { on } \Gamma_{f r}, \quad \mathbf{u} \cdot \mathbf{n}=0 \text { on } \Gamma_{f r}
$$

where $m$ is the stress exponent and $\beta$ is the friction coefficient. This viscous friction (or sliding) law is an entire model itself as it can represent bedrock heterogeneities, material properties change, an unclear interface, etc. Typically, in glaciology it aims to model the non-linear rheology of a subglacial sediment layer (through a parameter $m>1$ ) and the water pressure from underlying hydrology (typically setting $\beta=k N^{q}, q \geq 1$ and $N=\rho g h-P_{\text {water }}$ the effective pressure).

Also, there is no consensus on the value of $m$ (or $q$ ) a classical choice for ice-streams flows consists to set $m=3$, (and $q=1$, see e.g. [8]). In a nutshell, the friction coefficient $\beta$ is a priori non uniform and depends on various quantities (bedrock roughness, subglacial water pressure, sediment properties, etc) and requires to be accurately calibrated. The last section of the present paper focuses on this question.

The boundary conditions on the lateral boundaries are as follows. At inflow, we impose the vertical velocity profile as the shallow power-law uniform solution, see e.g. [19]; a lithostatic pressure is imposed at the outflow boundary. The boundary conditions on the lateral boundaries are as follow;. at inflow, we impose the vertical velocity profile as the shallow power-law uniform solution, see e.g. [20, 19]; at outflow, a lithostatic pressure is imposed.

\subsection{Numerical solution of the forward problem}

The numerical solution of the forward model (3)-(4) is obtained using the four field finite element scheme, of order two in space, and the augmented Lagrangian type algorithm detailed in [14]. Such a formulation arises when applying a splitting technique on a minimal dissipation form of the corresponding variational problem. 
The splitting, by introducing the strain-rate tensor $\underline{\boldsymbol{D}}$ as an unknown, allows the linear part and the non linear part of the governing equations to be solved sequentially. This algorithm provides important cpu-time saving compared to a classical fixed point approach as well as memory saving (which becomes important for solving the adjoint problem, as in Section 3). This formulation requires a three-field finite element discretization in order to handle the tensorial unknowns $\underline{D}$ and $\underline{S}$ in addition to the vectorial unknown $\mathbf{u}$ and the scalar unknown $p$ (see [21]). We refer to [14] and references therein for a complete assessement of this accurate and robust second order finite element scheme.

Free surface dynamic. Recall that $\Gamma_{s}$ is supposed to be the graph of a function $h(x, t)$, an Arbitrary Lagrangian Eulerian (ALE) formulation is adopted for the treatment of its dynamic. The mesh velocity is computed from the solution of an elastic problem or from a vertical homothety if appropriate. Equation (8) is solved using the characteristics method; it gives:

$$
\partial_{t} h+u_{x} \partial_{x} h=\left.\frac{d h}{d t}\right|_{\chi}=a+u_{z}, \text { RHS given }
$$

where $\chi$ denotes the characteristic curves. Then for each node, we obtain a Cauchy problem which can be solved using classical schemes (here Euler or Runge-Kutta order 2). In the present study, inverse rheological questions are investigated and the numerical experiments are only performed on steady state situations. The steadystate state is obtained by running in time the free-surface model until the average relative normal velocity (discrete 1-norm) at the vertices is lower than $10^{-4}$ and the relative variation of volume is lower than $10^{-5}$.

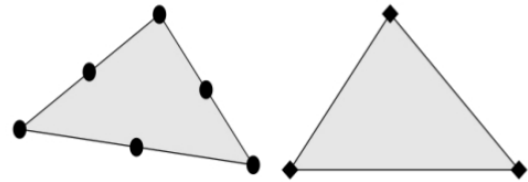

(a) $u_{h} \in\left(\mathbb{P}_{2}-\mathcal{C}^{0}\right)^{2}$ (b) $p_{h} \in \mathbb{P}_{1}-\mathcal{C}^{0}$

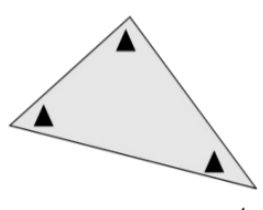

(c) $\tau_{h}$ and $d_{h} \in \mathbb{P}_{1}^{4}$
Figure 2: Four-field FEM implemented: extended Taylor-Hood mixed finite elements corresponding to a $P_{2}$ continuous velocity, $P_{1}$ continuous pressure and $P_{1}$ discontinuous strain rate and deviatoric stress tensors.

\section{Inverse model}

This section briefly describes the adjoint-based method used in the present work to investigate inverse questions (sensitivities, data assimilation - identification). The resulting numerical tool is accurate since gradients are of second order. Furthermore it is efficiently computed since based on the newly obtained differentiation of the four-field finite element solver presented previously.

\subsection{Cost function and optimization problem}

The output of the model is represented by the cost function $j$ (a scalar valued function) which depends on the parameters $k$ of the model. Given velocity observations denoted $u^{o b s}$, we define the cost function $j$ which measures the discrepancy between the computed variable (state of the system) and the available data at a given time. Extra terms can be added to the definition of the cost function to include $\mathrm{n}$ a priori on the physics of the control variables and/or a regularization of the optimization problem:

$$
j(\mathbf{k})=\int\left\|C u^{o b s}-\mathbf{u}(\mathbf{k})\right\|_{2}^{2} d \mathbf{x}+\text { regularization terms }
$$

where $\mathbf{u}$ is the computed velocity of the system and $C$ is the observation operator.

The optimization problem writes:

$$
\min _{\mathbf{k}} j(\mathbf{k})
$$

This minimization problem is solved numerically by the quasi-Newton local descent algorithm L-BFGS implemented in the M1QN3 routine (see [22]). To do so, the gradient of $j$ has to be computed. The corresponding procedure is described hereafter.

\subsection{Adjoint model and gradient accuracy}

The cost function gradient is deduced from the adjoint code of the forward direct code. The direct code, solving the power-law Stokes problem at order two in space, is written in Fortran 95. The development of the sensitivity and parameter identification modules are part of the DassFlow project which was originally designed for shallow-water models, see [23, 24, 25]. The present code, called DassFlow-Ice (or DassIce), has been developed during the $\mathrm{PhD}$ of the first author (see [26]) and is publicly available (see [27]).

There exists three approaches in order to obtain a solution algorithm of the adjoint problem. The most natural one called continuous appproach consists in analytically derive the continuous adjoint model and 
then discretized it with any appropriate numerical scheme. The main difficulty of this approach lies in the potentially difficult analytical derivation, sometimes requiring some simplifying assumptions to be achieved. Conversely, the discrete approach consists in deriving the adjoint model of the discrete formulation of the forward model. This method ensure a good consistency between the forward and the adjoint discrete models.

In DassFlow software, the adjoint model is obtained by using algorithmic differentiation of the source code (which is a particular case of the discrete approach). The principle of algorithmic differentiation is based on the idea that any numerical program is a sequence of elementary operations that can be analytically derived with the usual derivation rules.

Let us consider the forward code as an operator $\mathcal{M}$ : $\mathbb{R}^{n} \rightarrow \mathbb{R}^{s}$ that computes for a set of input parameters $\mathbf{k} \in \mathbb{R}^{n}$, an output vector $Y \in \mathbb{R}^{s}$. One denotes by $m_{k}$ an elementary operation and by $X_{k-1}$ the value of the variables at step $k$. If one includes the control vector $\mathbf{k}$ in the global set of variables for the program $\left(\mathbf{k} \subset X_{0}\right)$, one can write:

$$
Y=\mathcal{M}(\mathbf{k})=m_{p}\left(X_{p-1}\right) \circ m_{p-1}\left(X_{p-2}\right) \circ \ldots \circ m_{1}\left(X_{0}\right)
$$

The Jacobian matrix of $\mathcal{M}$ is then given by:

$$
\frac{\partial \mathcal{M}}{\partial \mathbf{k}}(\mathbf{k})=m_{p}^{\prime}(X p-1) \times m_{p-1}^{\prime}\left(X_{p-2}\right) \times \ldots \times m_{1}^{\prime}\left(X_{0}\right)
$$

where $m_{k}^{\prime}$ are the Jacobian matrices associated to the elementary operations $m_{k}$.

Tangent mode. In practice the Jacobian matrix (16) is too complicated to be computed and to heavy to be stored in memory. However one can compute a directional derivative associated to a given direction $\delta \mathbf{k}$ :

$$
\frac{\partial \mathcal{M}}{\partial \mathbf{k}}(\mathbf{k}) \cdot \delta \mathbf{k}=m_{p}^{\prime}(X p-1) \times m_{p-1}^{\prime}\left(X_{p-2}\right) \times \ldots \times m_{1}^{\prime}\left(X_{0}\right) \cdot \delta \mathbf{k}
$$

where the computation is performed from the right to the left by simple matrix-vector products. This method leads to obtain the linear tangent model by algorithmic differentiation.

Reverse mode. The remaining issue is that the linear tangent model is not appropriate to compute the gradient since it requires $s$ integrations (for a solution vector in $\mathbb{R}^{s}$ ). Automatic differentiation in reverse mode allows to compute the scalar product between the transposed of the Jacobian matrix (i.e. the adjoint model) and a vector $\widetilde{Y}$ :

$$
\left(\frac{\partial \mathcal{M}}{\partial \mathbf{k}}(\mathbf{k})\right) \cdot \widetilde{Y}=m_{1}^{\prime T}(X 0) \times m_{2}^{\prime T}\left(X_{1}\right) \times \ldots \times m_{p}^{\prime T}\left(X_{p-1}\right) \cdot \widetilde{Y}
$$

This expression is also computed from the right to the left by a sequence of matrix-vector product.

The automatic differentiation approach ensures a better consistency between the computed cost function and its gradient and a high accuracy of the computed gradient, since it is the computed cost function that is differentiated. A large part of this extensive task can be automated using automatic differentiation (see [28]).

The linear solver used is MUMPS ([29]) and the differentiation of the linear system solving process is achieved using a "bypass" approach which considers the linear solver as an unknown black-box (see [13]).

The adjoint code is derived using the automatic differentiation tool Tapenade (see [30]).

Let us notice that the continuous exact adjoint system of the power-law Stokes equations is presented in [31] for a general optimal control framework and in [13] for the problem with a nonlinear friction boundary condition (treated in the present work).

A single integration of the forward model (3)-(4) followed by a single integration of the adjoint model allow to compute all components of the gradient of the cost function. The computed gradient has been validated against order two finite differences and is adjustable in precision (from the fully incomplete gradient corresponding to the so called "self-adjoint" method in the glaciology community, to the exact order two accurate gradient) providing time and memory saving for an identical precision (see [13, 14]).

We consider in the following, as control variables, the two parameters of the rheological law which are the consistency $\eta_{0}$ and the power-law exponent $n$ (see (6)) and the two parameters of the friction law which are the friction coefficient $\beta$ and the exponent $m$ (see (11)). Therefore, we set $\mathbf{k}=\left(n, \eta_{0}\right)$ and we write the total differential $d j$ of the cost function $j$ as follows:

$$
d j(\mathbf{k})=\frac{\partial j}{\partial \eta_{0}}(\mathbf{k}) \cdot \delta \eta_{0}+\frac{\partial j}{\partial n}(\mathbf{k}) \cdot \delta n+\frac{\partial j}{\partial \beta}(\mathbf{k}) \cdot \delta \beta+\frac{\partial j}{\partial m}(\mathbf{k}) \cdot \delta m
$$

The output of the adjoint code corresponds to the partial derivatives of the cost function $j$ with respect to the 
chosen control variables.

Every variable in the control vector is only a potential control variable. In practice, it is possible to identify only a few of them simultaneously.

\subsection{Local Sensitivity Analysis}

Let us consider $s$ parameters to be controlled i.e. $\mathbf{k}$ is a vector value of size $s$. Then, the $s$ gradient value components, $j^{\prime}(\mathbf{k})=\left(\frac{\partial j}{\partial k_{i}}\right)_{1 \leq i \leq s}$, give the sensitivity of the output function $j$ with respect to the $i^{\text {th }}$ control component, at the point $\mathbf{k}$.

The sensitivity analysis allows to study how perturbations on the input parameters of a model lead to a perturbed output. The use of an adjoint model provides a local sensitivity analysis around a given point by computing the Fréchet derivative of the cost function $j$ (e.g. defined by (13)) with respect to the control variables k. This sensitivity analysis tool is an important feature which provides a better understanding of both the physics and the model by quantifying the roles of the various physical parameters and the influences of parameter variations on the behavior of the system. Since control parameters can be spatially distributed, the results can be sensitivity maps (e.g. sensitivity maps with respect to the consistency $\eta_{0}$ ).

\subsection{Data Assimilation and Twin Experiments}

The main goal of the present article is to investigate the sensitivities and inference capabilities of a variational method for geophysical free-surface flows with a power-law rheology, with respect to the rheological parameters and the basal properties. To do so, we design in next sections fully representative flows in term of regimes, and twin experiments including realistic noised surface observations are performed.

Twin experiments are defined as follows. The reference parameters of the model $k_{r e f}$ are used to generate observations $u_{o b s}$. Then, the goal is to retrieve the set of parameters $k_{\text {ref }}$ starting from an initial guess $\mathbf{k} \neq k_{\text {ref }}$ using the minimization of the cost function $j$. In order to avoid the so called inverse crime and to simulate realistic situations, a random Gaussian noise is added to the synthetic data obtained from the numerical model.

\subsection{On the efficiency of the adjoint solution based on the four-field finite element solver LA}

Since the adjoint code is obtained from sourceto-source automatic differentiation of the forward code, the performances of the adjoint computation are strongly linked to the algorithm considered for the solution of the forward problem. In the present case, automatic differentiation has been applied to the implementation of the augmented Lagrangian type algorithm called LA. It is described and assessed in details in [14].

The automatic differentiation of an iterative procedure is handled using a reverse accumulation technique. It consists in computing a partial derivative associated to each state encountered by the forward solver. The final adjoint state is then computed as the sum of the partial derivatives (as a consequence of the chain rule). This process a priori requires to store as many states of the system as iterations performed by the forward solver to reach the converged state. We address [13] for a comprehensive description of this approach.

This procedure applied to LA algorithm allows very good performances to be obtained compared to the derivation of a fixed point type algorithm. Firstly, an important time-saving is obtained according to the time-saving provided by $\mathbf{L A}$ algorithm for the solving of the forward problem. Secondly, the splitting considered in LA algorithm implies that the Stokes system is not modified along the iterations. It therefore requires only one factorized Stokes stiffness matrix to be stored. The non-linear tensor equation, discretized on a discontinuous finite element basis, is block-diagonal and therefore solved along the assembly. It follows that no storage of this matrix is required.

The present implementation allows to obtain for the complete solution (i.e. the forward solution plus the adjoint solution), compared to the fixed point approach used in [13], on a mesh of 300000 elements, a cpu-time ratio of 5 and a memory ratio of 4 . These ratios are smaller than one can expect, based on the ratio obtained for a single forward solve, due to a non-robust behavior of the Newton algorithm used to solve a non-linear scalar equation on the norm of the strain-rate within the algorithm (see [14]). The use of a more robust algorithm could allow to significantly improve these ratios.

In other respect, technical adjustements in terms of checkpointing can provide, using more memory, a better cpu ratio. 


\section{Assessment of the inverse numerical model: sur- face observations and near-surface singularity}

\subsection{Issue addressed}

The power-law form is an empirical fit to laboratory and field data within a finite shear rate range. But, this law leads to an infinite viscosity singularity for vanishing shear-rates (in the shear-thinning case, $n>1$, see equation (6)). In other respect, it has been demonstrated that this singularity might lead to an ill-posed direct problem if a free-surface is considered (see [16]).

In a geophysical fluid flows context, measurements of rheological parameters are difficult and may depend on the experimental setup, the shear-rate range and, for fluid such as ice or lava, on the temperature (see [8], [11], [32]). For instance, the laboratory conditions will generally differ from the field. It follows that the values for both the power-law exponent $n$ and the consistency $\eta_{0}$ are not clearly established.

In the glaciology context, and concerning the infinite viscosity singularity at the free-surface, in [33] for example, the author proposes a regularization at low shear-rate by adding a constant (i.e. a Newtonian threshold). In [17] the authors consider the Glen's power-law (without low shear rate regularization and using $n=3$ ), a locally perturbated topography (around a mean slope) and a friction condition at the bottom. Using shallow asymptotic expansions (i.e. with respect to the aspect ratio $\varepsilon$ ) and matching techniques between the near-surface layer and the "bulk" solution (far from the free surface), the authors give explicit expressions of the stress components and velocity fields at the free surface. At the leading order (first order in $\varepsilon$ ), the stress components $\sigma_{x x}, \sigma_{x z}$ are unphysically singular. They show that the singularity appears in a boundary layer of size $O\left(\varepsilon^{1 / 3}\right)$, which might not be negligible in practice.

The authors show nonetheless that at first order in $\varepsilon$, the singular solution remains "passive", in the sense that the free-surface geometry may not be changed because of the presence of the boundary layer. In other words, for shear-thinning shallow flows, one should be able to infer basal behavior from surface velocities, using a power-law model without any (non physical) regularization at low shear rate.

Since surface velocities may be used to invert the fluid properties studied, since all inversions on the considered geophysical flows are based on surface observations, it seems to be relevant to assess the present inverse approach inferring capabilities "through" this singular boundary layer. It is what we propose to numerically assess in the experiments below.

\subsection{Methodology of investigation}

In order to address this question, given horizontal surface velocity observations, we compute sensitivities with respect to a locally defined power-law exponent $n$. It means that the solving of the adjoint code provides a gradient of the cost function with respect to $n$ around a constant value $n=3$.

Test case design. We consider the flow occuring in a non uniform slab with a perturbated sinusoidal bottom. The shape of the bottom is built as a carrier sinusoidal function $b_{0}$ perturbed with two higher frequencies $b_{1}$ and $b_{2}$. The carrier wave has an amplitude $a_{0}=2 / 5 \bar{h}$ with $\bar{h}=1000 m$ the average thickness and the two perturbations have an amplitude $a_{1}=a_{2}=1 / 5 \bar{h}$. The frequency of the carrier wave is $f_{0}=5 \bar{h}$ and the two perturbations have frequencies $f_{1}=2 \bar{h}$ and $f_{2}=\bar{h}$. This topography has been built according to the results given in [13] providing the fact that, using dense surface velocity observations with a $1 \%$ noise, the basal variability frequency transmits to the surface up to two thicknesses; higher frequencies does not a priori affect the surface. The computational domain presents an aspect ratio of $1 / 10$ on a $2 \%$ slope. The inflow on the left boundary is given by the analytical Poiseuille type solution of the power-law uniform stationnary flow (see e.g. [13]). The boundary condition at outflow is a prescribed lithostatic (ice) pressure. The simulation is run in time until a steady state is reached. The resulting stationnary free-surface flow is thus obtained.

Considered flow regimes. Two different regimes are considered in the following sensitivity experiments corresponding to a low sliding and a rapid sliding at the bottom. The level of sliding is defined using the slip ratio $r$ which quantifies the relative contributions of the viscous deformation and the sliding on the surface velocities. It is defined by:

$$
r=\frac{\overline{u_{b}}}{\overline{u_{s}}-\overline{u_{b}}}
$$

where $\overline{u_{b}}$ and $\overline{u_{s}}$ represent respectively the average velocity at the bottom and the surface. In what follows, the low sliding case corresponds to an average slip ratio $r_{l}=0.05$ which leads to surface velocities of $1 \mathrm{~m} / \mathrm{y}$ (meter per year) to $10 \mathrm{~m} / \mathrm{y}$ typical of an ice 
sheet regime; $95 \%$ the surface velocity comes from the viscous deformation of the ice. The rapid sliding case corresponds to an average slip ratio $r_{r}=20$ which leads to surface velocities of $50 \mathrm{~m} / \mathrm{y}$ to $500 \mathrm{~m} / \mathrm{y}$ typical of an ice stream regime; $95 \%$ of the surface velocity comes from the basal sliding. A linear sliding law, corresponding to $m=1, m$ defined by (11), is considered. Surface velocity data $u_{s}^{o b s}$ are generated for these two situations and a cost function is defined as:

$$
j(n)=\int_{\Gamma_{s}}\left\|u_{s}(n)-u_{s}^{o b s}\right\|_{2}^{2} d \mathbf{x}
$$

The approximation of a near-surface singularity is assessed using two different meshes; an isotropic one and mesh refined at the surface. The surface refinement allows to converge to the singularity as the size of the element goes to zero. Hereafter, a "boundary layer" of $1 / 5$ of the average thickness $\bar{h}$ is discretized with an element size ratio of 10 compared to the elements in the bulk.

\subsection{Numerical results}

Figures 3 and 5 plot the resulting sensitivity of the model with respect to the power-law index $n$ defined by element $\partial j / \partial n\left(u_{o b s}, n_{0}\right)$ using the cost function (21) for both meshes and the two different flow regimes ( $r=0.05$ and $r=20$ respectively). The corresponding shear-rates $\dot{\gamma}$ (see equation (7)) are plotted in Figures 4 and 6 for both slip ratios. It highlights the strong yet varying correlation between $\dot{\gamma}$ and the computed gradient.

In all the following plots, the bounds of the colorbars have been modified to match one another in order for the figures to be compared. The values displayed under the color bars as min and max give the original bounds of the plotted field. Plots have been cut on the sides to remove the sensitivity values close to the lateral boundaries, particularly the Dirichlet boundary $\Gamma_{l}$, which are not representative of the variations sought since the lateral boundary conditions are fixed.

Analysis of the low sliding case $(r=0.05)$. The case of strong friction at the bottom shows a relatively layered repartition of the sensitivity $\partial j / \partial n$ from high at the bottom to low at the surface for both meshes (see Figures 3(a) and 3(b)). These sensitivity maps are strongly related to the shear-rate fields which are naturally decreasing from the bottom to the surface

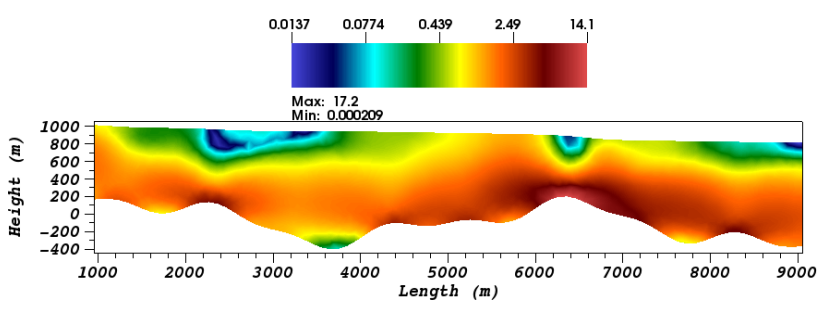

(a) Isotropic mesh

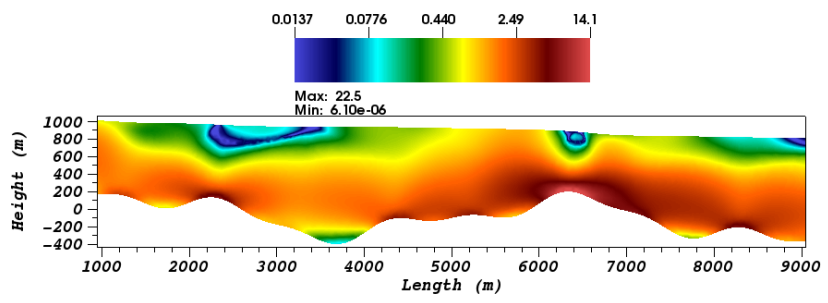

(b) Near-surface refined mesh

Figure 3: Slip-ratio $r=0.05$ : Local sensitivity $\partial j / \partial n$ computed around a state $n_{0}=2.25$ using observations $u^{o b s}$ obtained with $n=3$. The color scale is logarithmic. The gradient values, constant by element, have been normalized by the area of their element in order to remove the weight the element size induces (which is a normal feature in an optimization perspective but prevents from drawing a readable sensitivity map on an anisotropic mesh).

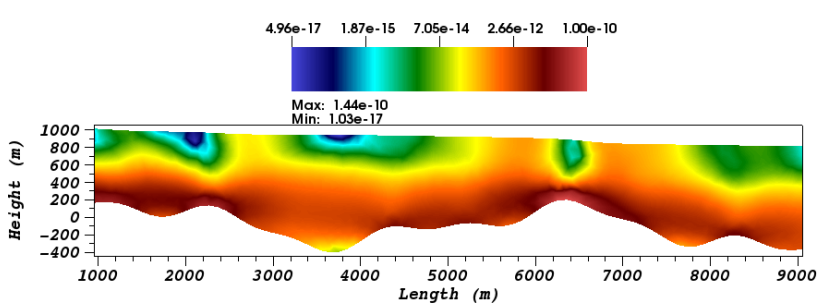

(a) Isotropic mesh

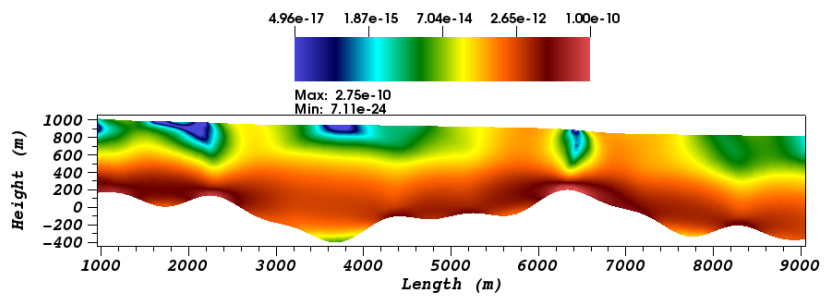

(b) Near-surface refined mesh

Figure 4: Slip ratio $r=0.05$ : Computed shear-rate $\dot{\gamma}$ for the observed state (with $n=3$ ) in $\mathrm{s}^{-1}$. The color scale is logarithmic.

(due to the strong friction condition which induces a non-linear Poiseuille flow, see Figures 4(a) and 4(b)). The mesh refinement at the surface approximates the infinite viscosity but the discretization induces a cut-off on the shear-rate that depends on the size of the elements. The convergence to the viscosity singularity is clear since the minimum shear-rate obtained on the 


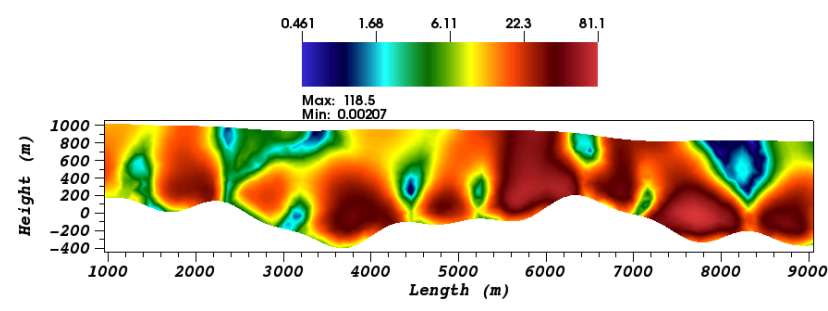

(a) Isotropic mesh

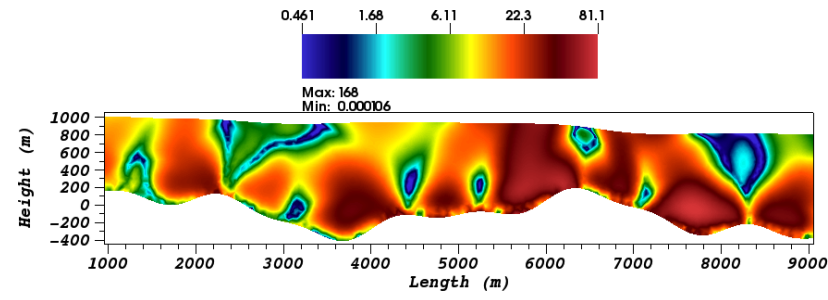

(b) Near-surface refined mesh

Figure 5: Slip-ratio $r=20$ : Local sensitivity $\partial j / \partial n$ computed around a state $n_{0}=2.25$ using observations $u^{o b s}$ obtained with $n=3$. The color scale is logarithmic. The gradient values, constant by element, have been normalized by the area of their element in order to remove the weight the element size induces (which is a normal feature in an optimization perspective but prevents from drawing a readable sensitivity map on an anisotropic mesh.

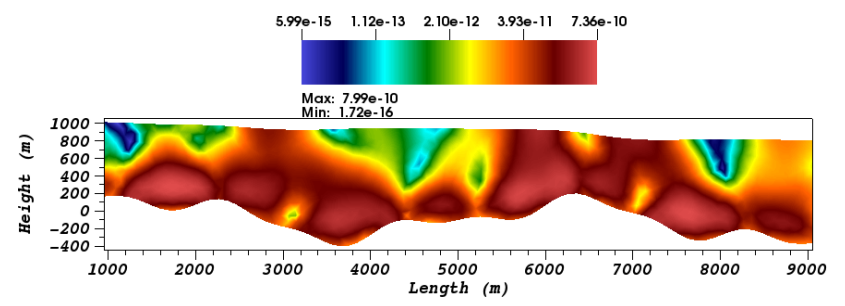

(a) Isotropic mesh

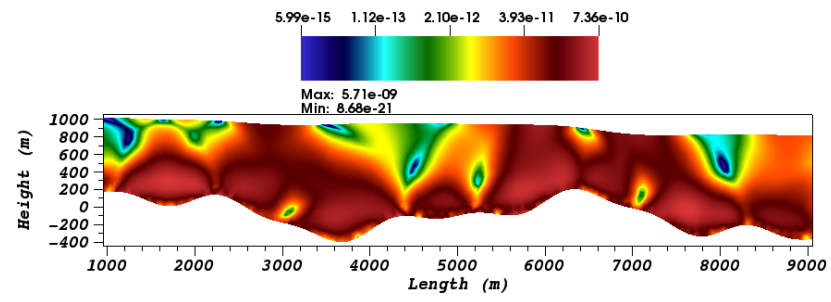

(b) Near-surface refined mesh

Figure 6: Slip ratio $r=20$ : Computed shear-rate $\dot{\gamma}$ for the observed state (with $n=3$ ) in $\mathrm{s}^{-1}$. The color scale is logarithmic.

refined mesh is much smaller than the one obtained on the isotropic mesh. However, both the gradient and the shear-rate distribution look identical for both meshes. It follows that a good convergence of the solution was achieved on the coarser mesh and that the refinement of the singularity does not affect the solution. In particular, the singularity remained passive in terms of normal surface velocities in the transient simulation since the steady state free surfaces are identical for both meshes.

Analysis of the rapid sliding case $(r=20)$. In the case of rapid sliding (Figure 5 and 6), the correlation between the shear-rate and the sensitivity is less clear. The presence of a rapid sliding allows high shear rates to develop close to the surface and the layered repartition of the shear-rate (from high shear-rate at the bottom to low shear-rate at the surface) observed for low sliding is no longer observable. Alongside this aspect, the sensitivities $\partial j / \partial n$ show non trivial low sensitivity zone in highly sheared area.

However, as well as the case of low sliding, both meshes show an identical result for the sensitivities $\partial j / \partial n$. The refined boundary layer does not affect the sensitivity map leading to the same conclusions on the role of the singularity. We point out that the higher absolute values for the gradients in the case of rapid sliding, compared to the low sliding case, are only due to higher surface velocities and therefore a higher misfit.

The observed independance of the solution and the gradient with respect to the power-law exponent to the surface velocities numerically assess the robustness of such data to the viscosity singularity.

Based on these results, the next sections focus on the inference of the rheology and basal properties of this type of flow based on surface velocity observations.

\section{Virtual Rheometry: constant power-law expo- nent identification}

The present identification is performed on a radarsensed topography of an ice flow with a complex bottom shape but the results can be extended to general experimentally controlled flows with simpler bottoms.

The computational domain considered is built from the radar-sensed topography and bedrock of the Mertz ice-tongue in East Antarctica measured along a flowline (American program ICECAP 2010, see [34]). It is plotted in Figure 7. The present study focuses on the grounded part of the glacier which has an average thickness of $1 \mathrm{~km}$. The simulation is identical (in terms of boundary conditions) to the one presented in Section 4.2. the friction coefficient $\beta$ is adjusted accordingly to the two flow regimes (corresponding to average slip 


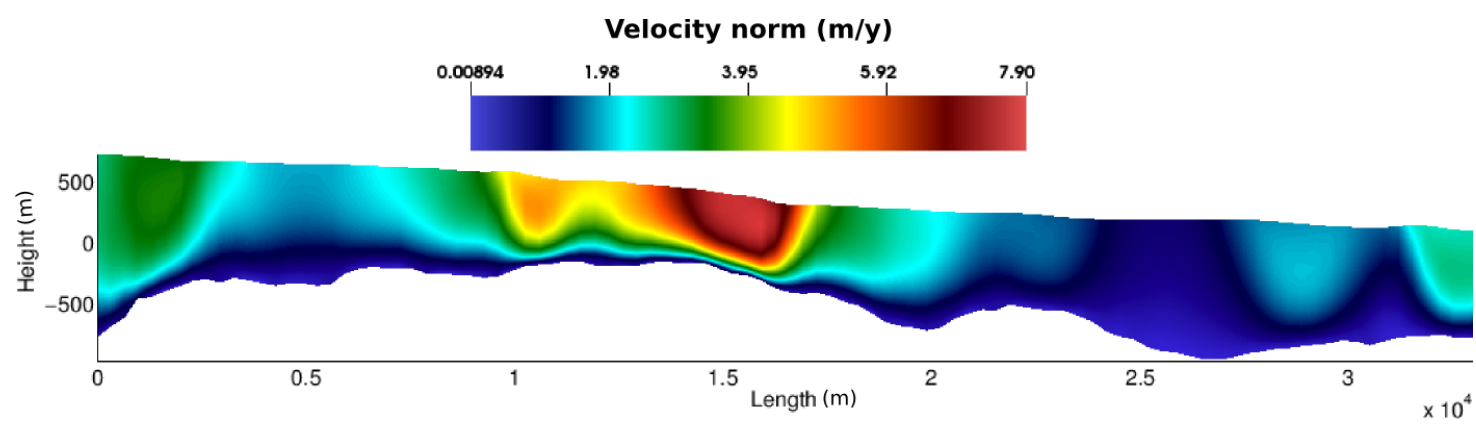

Figure 7: Vertical cut of the outlet glacier Mertz, Antarctica (topography profile from ICECAP 2010 within Ice bridge, provided by B. Legrésy, LEGOS, France), $\mathrm{x}$-scale $=2 / 5$. The norm of the velocity is plotted in the low sliding case $(r=0.05)$. The mesh is made of 4000 triangular cells on approximatively 10 layers.

ratios $r=0.05$ and $r=20)$.

From the results of the previous section, considering the reliability of surface velocity observations, we propose hereafter to use the data assimilation as a complementary numerical tool to support rheometrical investigations of power-law fluids such as laboratory measurements (see e.g. [32]) or estimation from real context data (see e.g. [35]).

We then present identification results of the scalar valued power-law exponent $n$ using noisy synthetic data. These numerical experiments lead to what can be called a virtual rheometer. The cost function is defined by:

$$
j(n)=\int\left\|u_{s}(n)-u_{s}^{o b s}\left(n_{t}\right)\right\|_{2}^{2} d \mathbf{x}
$$

Since a scalar value is identified, there is no need for regularization. Likewise, the adjoint model is unnecessary for identifying a scalar value but is used anyway.

Two data sets with Gaussian noise of $10 \%$ and $50 \%$ are considered for the experiments. For both data sets, the decreasing of cost and gradient as well as the evolution of the power-law index value along the data assimilation process are plotted in Figure 8 for the case $r=0.05$ using as a first guess $n_{0}=1$. The case of rapid sliding $(r=20)$ provides identical results and is consequently not plotted.

Firstly, the almost linear decreasing of cost and gradient (on a logarithmic scale) for each situation demonstrates the robustness of the identification of a scalar value. What is also of importance is the extremely large decreasing of the gradient. As a matter of fact, the gra-
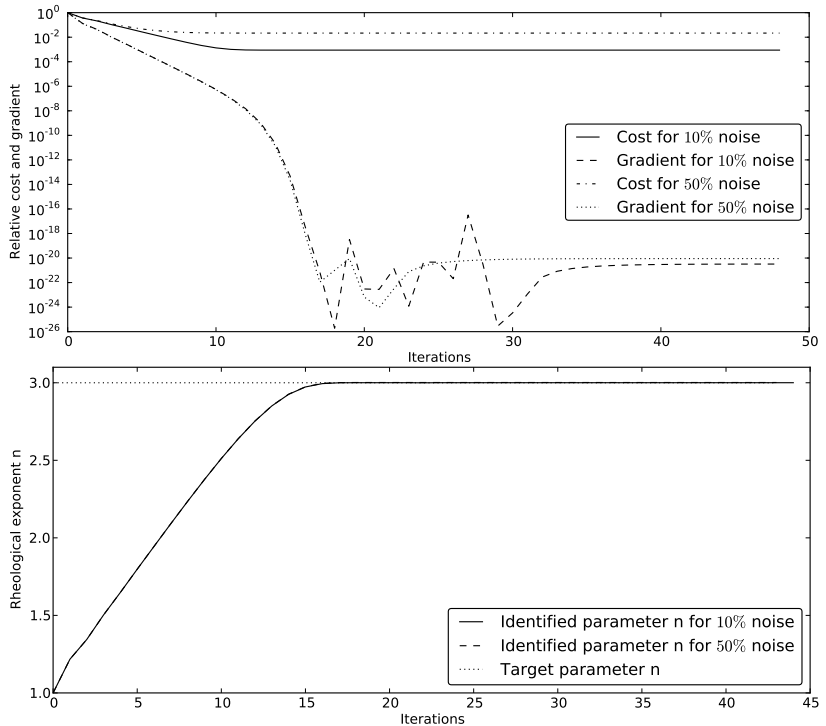

Figure 8: Slip ratio $r=0.05$ : relative cost and gradient during the data assimilation cycle for different levels of noise. Identified $n$ along the data assimilation cycle. Relative errors $\varepsilon_{n}=\frac{\left|n-n_{t}\right|}{n_{t}}$ (with $n_{t}=3$ the target) are: $\varepsilon_{n}=0.011 \%$ and $\varepsilon_{n}=0.055 \%$ for $10 \%$ and $50 \%$ of noise respectively

dient decreases by (more than 20 orders of magnitude). This is due to the fact that the model is highly sensitive to the rheological exponent $n$.

As can be see in Figure 8, the final calibrated value $n$ is very close to the target value $n_{t}$ even for $50 \%$ perturbed surface velocities. Once the target value is reached, the gradient norm quickly drops to a very small value making clear that the optimum has been reached. It follows that velocity data with large uncertainties are sufficient to recover the uniform value of $n$.

The present inverse tool can be very useful when 
rheometric measurements are more difficult to access than velocities, or simply inaccessible, even for poorly observed velocities such as e.g. extraterrestrial rheology estimation, see [36].

\section{Virtual rheometry: identification of $\eta_{0}$ (tempera- ture dependency)}

As we have seen in the previous section, surface velocities represent a robust information to characterize the rheology of a uniform stationary flow. The present approach for the characterization of the power-law exponent led to a virtual rheometer. The other major component of a power-law description is the consistency $\eta_{0}$ (see equation (6)), which allows to characterize the non-homogeneity of the fluid. It is related, in the case of ice or lava, to spatially non uniform coupled physical effects such as the composition (ice fabric or lava crystal fraction) or thermal physics, hence difficult to assess.

This section focuses on the sensitivity analysis of the steady-state model with respect to a thermal-dependent consistency $\eta_{0}(T)$ in order to observe and assess the role the temperature plays in the flow in terms of surface velocity through this parameter. This context is taken as an example of fluids where the spatial variability (through the temperature-dependency) is expected to be strongly influent.

Then, following the idea of a virtual rheometer, identifications of this distributed parameter are performed using synthetic data for a noise level of $1 \%$.

\subsection{Description of the Thermal Dependent Flow}

As well as lava, ice is considered as a pseudoplastic (shear-thinning) fluid. It is described by a power-law constitutive law called Glen's flow law which is generally considered to be temperature-dependent. In the case of a non-isothermal glacier, the temperature field is generally obtained using a coupled thermo-mechanical model for the ice. In the sequel, a steady-state temperature profile with a zero accumulation term, which corresponds to a linearly decreasing temperature $T$ with respect to the height $\bar{z}$ is considered (see e.g. [8]):

$$
T(\bar{z}, \lambda)=T_{s}-\lambda \bar{z}
$$

with $T_{s}$ the surface temperature and $\lambda$ the temperature gradient. It can take a large range of values. In order to study the effect of the temperature description of the consistency, a large temperature gradient $\lambda=0.04 \mathrm{~K} / \mathrm{m}$ corresponding to a difference of $40^{\circ} \mathrm{C}$ between the surface and the bottom for a $1 \mathrm{~km}$ thick glacier is considered hereafter. The surface temperature is therefore $T_{s}=233.15^{\circ} \mathrm{K}$.

The consistency $\eta_{0}(T)$ is obtained from the thermal law given in [8] written as follows:

$$
\eta_{0}(T)=\eta_{r} e^{\frac{-Q}{R T}}
$$

where $\eta_{r}$ is the reference viscosity, $R$ is the gas constant and $Q$ stands for an activation energy. In glaciology, the power-law exponent $n$ is generally taken equal to 3 and the values of $\eta_{r}$ and $Q$ are given (see [8] for ice and [11] for lava).

The range of temperature within the fluid can be much higher for lava but the definition and parameterization of the law leads to similar consistency gradients.

The numerical experiments aim to compare, for a given situation of sliding, an isothermal flow and a thermal dependent one. The isothermal runs are identical to those considered in Section 4.2 and the thermal dependent ones use a consistency $\eta_{0}$ described by relation (24). Again, every computational domain correspond to the free-surface flow at steady state. Four situations are therefore obtained corresponding to the two cases of sliding ( $r=0.05$ and $r=20)$ with and without a thermal description of the consistency.

Firstly sensitivity analyses are presented for the isothermal and the thermal dependent case for a given slip ratio. Identifications of the thermal dependent consistency are then performed.

\subsection{Sensitivity with respect to $\eta_{0}$}

Situations of low and rapid sliding at the bedrock are compared hereafter. It can make an important difference in terms of sensitivity with respect to $\eta_{0}$ by influencing the flow regime. Figures 9 and 10 plot the computed shear-rate for low sliding $(r=0.05)$ and rapid sliding $(r=20)$ respectively, with and without a temperature-dependent consistency.

For both situations of sliding, the use of a temperature gradient to compute $\eta_{0}$ (see (24)) leads to the appearance of a distinct highly sheared layer close to the bottom (due to a higher temperature and thus a 


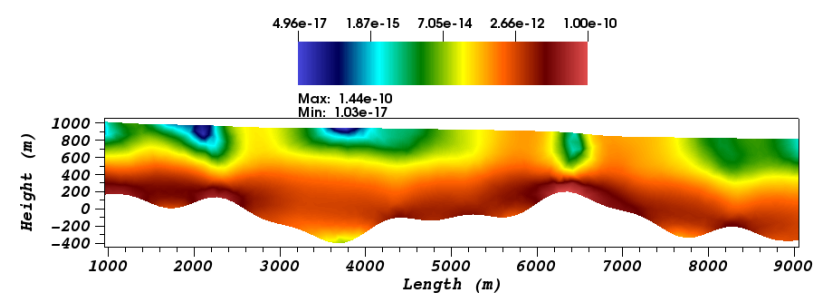

(a) Isothermal fluid

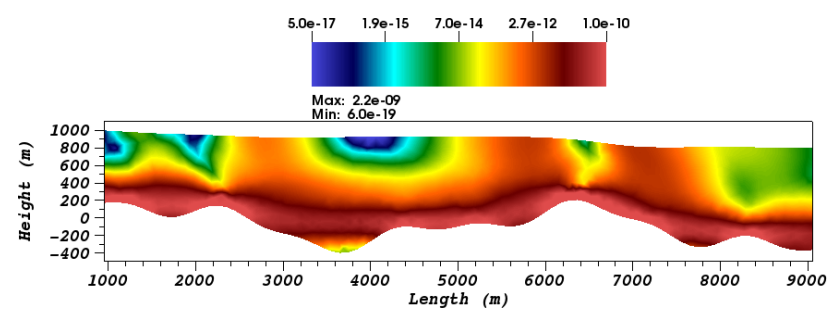

(b) Thermal dependent consistency $\eta_{0}$

Figure 9: Slip ratio $r=0.05$ : Computed shear-rate $\dot{\gamma}$ for the observed state (with $n=3$ ) in $\mathrm{s}^{-1}$. The color scale is logarithmic.

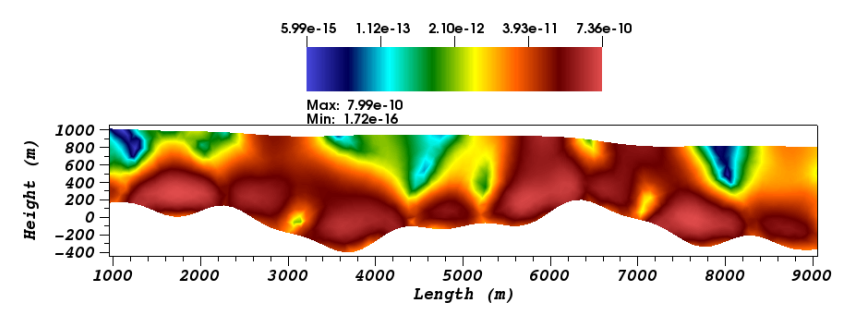

(a) Isothermal fluid

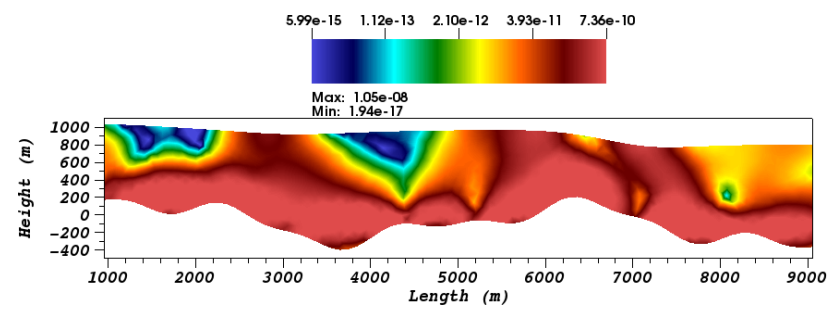

(b) Thermal dependent consistency $\eta_{0}$

Figure 10: Slip ratio $r=20$ : Computed shear-rate $\dot{\gamma}$ for the observed state (with $n=3$ ) in $\mathrm{s}^{-1}$. The color scale is logarithmic.

smaller viscosity). At the surface, the areas of higher shear-rate inbetween those of very low shear-rate are larger and of higher absolute value. Conversely, the very low shear-rate spots close to the surface are more localized and of smaller absolute value.

Figure 11 plots the sensitivity to the consistency $\eta_{0}$, in the case of rapid sliding $(r=20)$, around a reference value $\eta_{0}^{m}=\eta_{0}\left(T_{m}\right)$ which corresponds to a consistency field evaluated for a constant temperature $T$ taken equal to the average temperature of the original thermal dependent flow $T_{m}=-20^{\circ} \mathrm{C}$.

Similarly to the sensitivities with respect to the power-law exponent $n$, a correlation between the distribution of the gradient $\partial j / \partial \eta_{0}$ and the shear-rate map appears. However, the presence of the thermal physics make this correlation more vague; non trivial high sensitivity areas appear close to the surface when a thermal dependent consistency is used to generate the data. Conversely, the appearance of a high shear rate close to the bottom does not lead to a corresponding high sensitivity. The sensitivity maps, in the case of low sliding $(r=0.05)$, differ from Figure 11 but does not provide any additional insights on the influence of the thermal dependency in terms of surface velocities and is thus not plotted.

We point out that the differences in the steady geometries of the surface with or without the thermal dependency are very small, even though a stiffening of the near surface fluid occurs.

\subsection{Identification of $\eta_{0}$ based on surface velocity $o b$ - servations}

The following section is dedicated to the identification of a consistency field $\eta_{0}^{t}(T)$ computed using the linear thermal relation (24) with a temperature field obtained with $\lambda=0.04$ and plotted in Figure 12. The computational domain and flows are identical to Section 5, i.e. the real Mertz glacier topography with either slow $(r=0.05)$ or rapid sliding $(r=20)$, but using a consistency $\eta_{0}$ computed from (24).

Cost function and initial guess. The cost function used for the identification is defined by:

$j\left(\eta_{0} ; \gamma_{1}, \gamma_{2}\right)=\int_{\Gamma_{s}}\left\|u_{s}^{o b s}-u_{s}\left(\eta_{0}\right)\right\|_{2}^{2} d \mathbf{x}+\mathcal{T}\left(\nabla \eta_{0} ; \gamma_{1}, \gamma_{2}\right)$

where the synthetic data $u_{s}^{o b s}$ are the horizontal surface velocities perturbed by a $1 \%$ random Gaussian noise (the lack of robustness of these experiments lead us to consider a relatively small noise compared to the one used previously in the power-law index identification). The Tykhonov's regularization term $\mathcal{T}\left(\nabla \eta_{0}\right)$ controls the oscillations of the control variable gradient. It is defined by:

$\mathcal{T}\left(\nabla \eta_{0} ; \gamma_{1}, \gamma_{2}\right)=\gamma_{1} \int_{\Gamma_{s}}\left\|\partial_{x} \eta_{0}\right\|_{2}^{2} d \mathbf{x}+\gamma_{2} \int_{\Gamma_{s}}\left\|\partial_{z} \eta_{0}\right\|_{2}^{2} d \mathbf{x}$ 


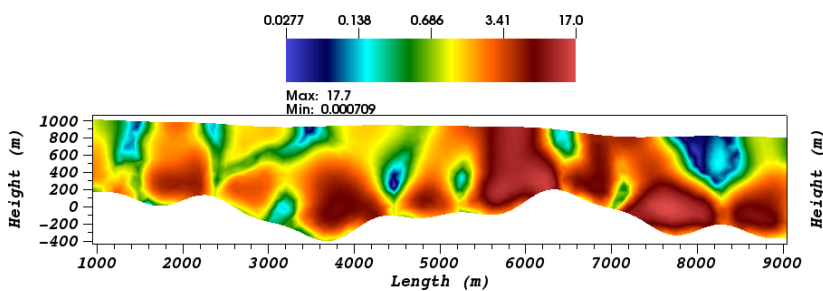

(a) Isothermal fluid

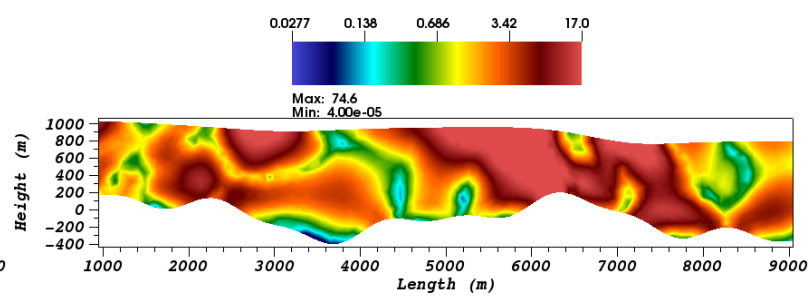

(b) Thermal dependent consistency $\eta_{0}$

Figure 11: Glacier flow with $\mathbf{r}=\mathbf{2 0}$ : sensitivity with respect to the consistency $\left(\partial j / \partial \eta_{0}\right)\left(\eta_{0}^{s}\right)$ around the state $j\left(\eta_{0}^{m}\right)$ (i.e. for an isothermal fluid at $-20^{\circ} \mathrm{C}$ ) for an isothermal fluid $\left(\eta_{0}\right.$ constant) and a thermal dependent one ( $\eta_{0}$ defined by $\left.(24)\right)$.

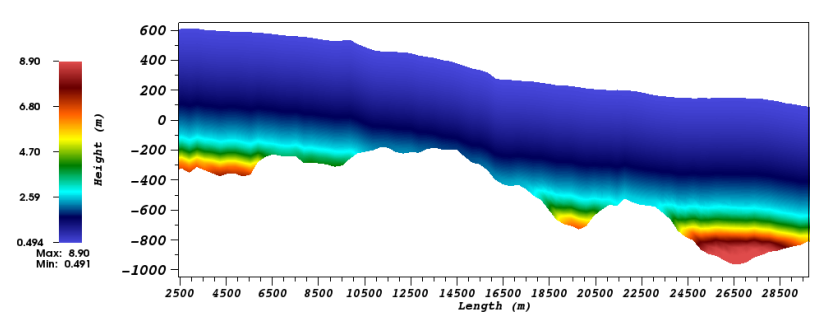

Figure 12: Target consistency field $\eta_{0}^{t}$ (in MPa.a ${ }^{1 / 3}$ ) computed from (24) and (12) with $\lambda=0.04$ in

where the parameters $\gamma_{1}$ and $\gamma_{2}$ quantify the strength of the imposed smoothness. The adjustment of these weights controls the degree of smoothness sought on the control variable. A classical approach, referred to as the Morozov's discrepancy principle, consists of choosing $\gamma_{1}$ and $\gamma_{2}$ such that the final cost $j\left(\eta_{0}^{f} ; \gamma_{1}, \gamma_{2}\right)$ matches the cost $j\left(\eta_{0}^{T} ; 0,0\right)$ with the perturbed $u_{s}^{o b s}$ (see e.g. $[37,13])$.

A layered consistency field decreasing from the surface to the bottom is sought. Therefore the balance on the gradients in both directions, appearing in the regularization term (26), is achieved using $\gamma_{1}=\gamma_{2} / 7$ (which corresponds to the exact ratio of the target consistency field). The exact ratio of 7 is not needed, but a very good guess (typically between 5 and 10) is required to achieve a decent identification.

Numerical results. The identification results are plotted in Figure 14 and 15 for low and rapid sliding respectively. For each case, two inferred $\eta_{0}^{f}$ are plotted corresponding to two different initial guesses which are $\eta_{0}^{i, m}=\eta_{0}\left(T_{m}\right), T_{m}=-20^{\circ} \mathrm{C}$ the average temperature and $\eta_{0}^{i, g}=\eta_{0}\left(T_{g}\right), T_{g}=T(\bar{z}, 0.02)$ with $T_{s}=-30^{\circ} \mathrm{C}$ (see equation (23)). Evolutions of the cost function and the gradient along the minimization procedure for the two different initial guesses are plotted in Figure 13.

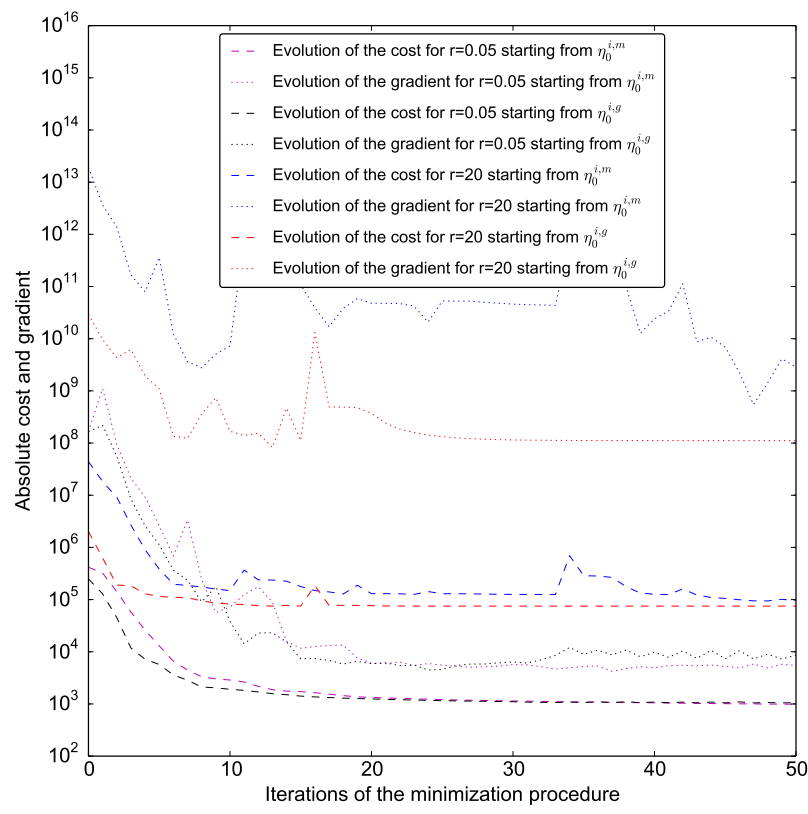

Figure 13: Costs and gradients along the minimization cycle. They are plotted in absolute value in order to compare the results of the two different initial guesses $\eta_{0}^{i, m}$ and $\eta_{0}^{i, g}$

From the evolution of the cost and the gradient, we can see that the behavior is more robust in the situation of moderate sliding $(r=0.05)$ for both intial guesses. In the case of rapid sliding $(r=20)$, the best intial guess $\eta_{0}^{i, m}$ leads to a clearly better result (smaller cost and smaller gradient). The difference between the two intial guesses in the case of moderate sliding is not really significant. This observation is retrieved in the relative errors in Table 1. However, the most important information we have from Figure 13 is the fact that the decreasing of the gradient, regardless of the level of sliding or the initial guess, is much smaller (3 to 4 orders of magnitude) than for the identification of the rheological exponent $n$ (20 orders of magnitude, 


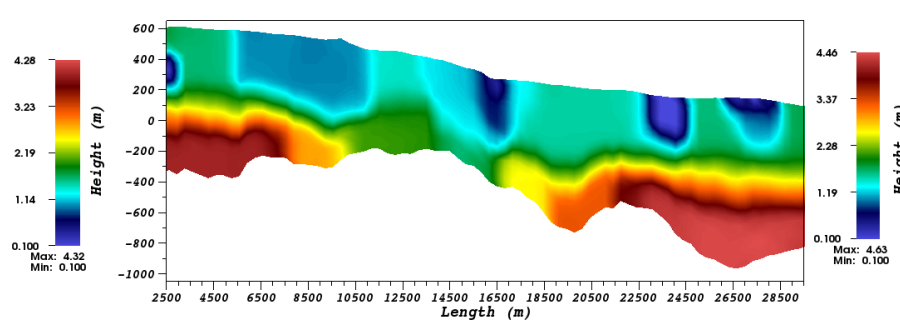

(a) Initial guess $\eta_{0}^{i, m}$

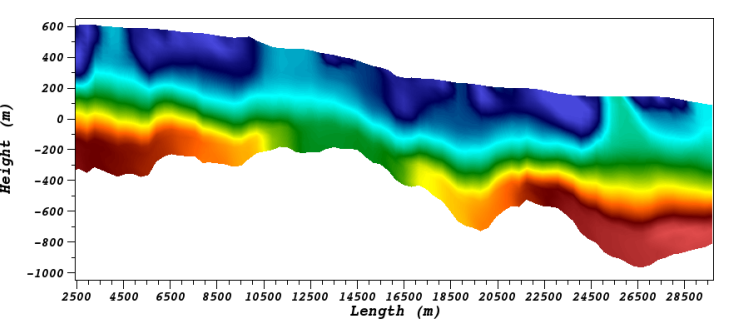

(b) Initial guess $\eta_{0}^{i, g}$

Figure 14: Slip ratio $\mathbf{r}=\mathbf{0 . 0 5}$ : Identified field $\eta_{0}^{f}$ in MPa.a ${ }^{1 / 3}$. A lower bound of 0.1 on the consistency has been imposed to prevent the minization procedure from generating negative values for $\eta_{0}$.

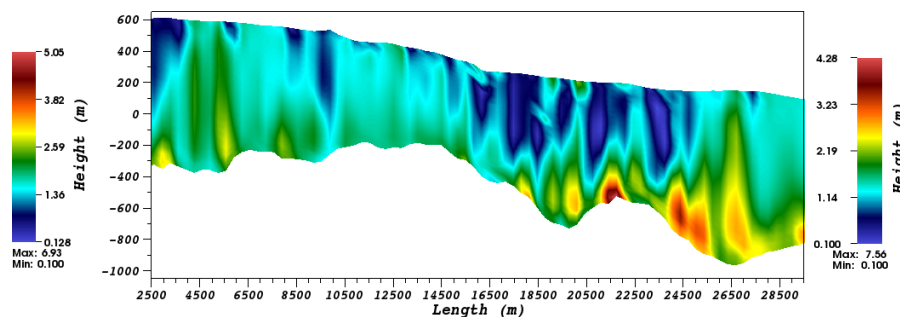

(a) Initial guess $\eta_{0}^{i, m}$

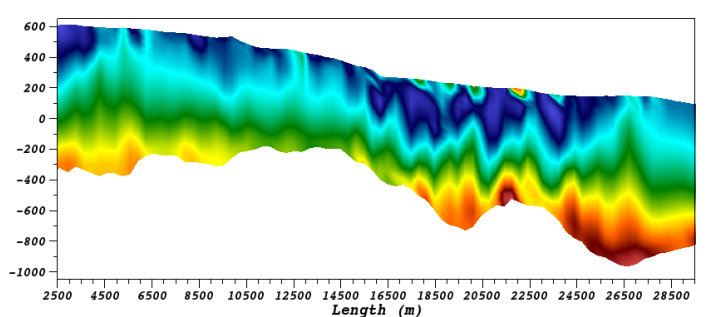

(b) Initial guess $\eta_{0}^{i, g}$

Figure 15: Glacier flow with $\mathbf{r}=\mathbf{2 0}$ : Identified field $\eta_{0}^{f}$ in MPa. $\mathrm{a}^{1 / 3}$. A lower bound of 0.1 on the consistency has been imposed to prevent the minization procedure from generating negative values for $\eta_{0}$.

see Figure 8$)$ or the identification of the $(\beta, n, m)$ triple (10 to 30 orders of magnitude, see Figure 17(a)). It brings to light the ill-posedness of the consistency identification problem.

The relative errors are given in Table 1. It clearly appears that the identification is easier in the case of low sliding. A small slip ratio represents a large contribution of viscous deformation to the surface velocities in favor of the identification of a rheological quantity whereas a higher slip ratio leads to a smaller contribution of the creep and of the rheology on the surface velocities.

It also makes sense in the light of the above sensitivities; the layered aspect of the sensitivity map in the case of low sliding (due to an important gradient of shear close to the bottom) helps to retrieve a layered consistency field. In the case of rapid sliding the distribution of the sensitivity is non trivial and so is the inferred consistency.

Discussion. Despite very good prior physical knowledges introduced in the identification process (by using the exact ratio between horizontal and vertical

\begin{tabular}{|c||c|c|c|c|}
\hline \multicolumn{1}{|c||}{ Slip ratio } & \multicolumn{2}{c|}{$\mathbf{r = 0 . 0 5}$} & \multicolumn{2}{c|}{$\mathbf{r}=\mathbf{2 0}$} \\
\hline Initial guess & Initial error & Final error & Initial error & Final error \\
\hline$\eta_{0}^{i, m}$ & $63.90 \%$ & $40.57 \%$ & $63.90 \%$ & $54.12 \%$ \\
\hline$\eta_{0}^{i, g}$ & $45.28 \%$ & $35.49 \%$ & $45.28 \%$ & $41.31 \%$ \\
\hline
\end{tabular}

Table 1: Relative error $\frac{\left\|\eta_{0}^{f}-\eta_{0}^{t}\right\|_{2}}{\left\|\eta_{0}^{t}\right\|_{2}}$ of the reconstructed rheological constant for noise level $\delta=1 \%$.

consistency gradient, good initial guesses and a perfect knowledge of all the other physical parameters) and considering the noise level on the surface data of $1 \%$, the resulting inferred consistency fields are poor. The basic layered aspect of $\eta_{0}^{t}$ is retrieved, thanks to the anisotropic regularization, but the range is quite different from the target one and the presence of a rapid sliding significantly deteriorates the inferred consistency. As one can expect, the use of $\eta_{0}^{i, g}$ as a first guess leads to a better identified consistency (see Table $1)$.

This result represents a major component of data assimilation that can be called equifinality of the system; an identical end state can be reached by different sets of parameters. In this situation, defining the end state as the horizontal surface velocities, $\eta_{0}^{t}$ and $\eta_{0}^{f}$ lead to the same ending. It means that the inverse problem is 


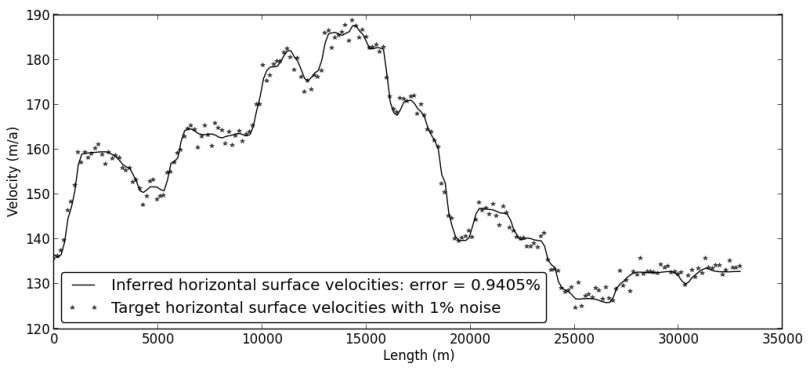

Figure 16: Normalized horizontal surface velocities: the plain line represents the computed surface velocities after identification and the stars represent the target surface velocities of the glacier flow considered in section 6.3 perturbed by a $1 \%$ Gaussian noise. The error is the relative error between both sets.

ill-posed.

The present inverse results show that an uncertainty on the consistency do not greatly affect the bulk flow. Its inference is a strongly ill-posed problem. The target and inferred surface velocities are plotted in Figure 16 in the case of rapid sliding $(r=20)$ with $\eta_{0}^{i, m}$ as a first-guess (which is the situation producing the larger relative error on the inferred consistency) to highlight the equifinality aspect.

Although physically questionable (in terms of dimensions of the problem), identifications of a spatially varying power-law exponent $n$ on the same situations lead to relative error of $0.076 \%$ and $1.86 \%$ on the low sliding and rapid sliding case respectively (with an initial guess $n=1$ ), confirming the greater robustness of the identification of $n$.

These results suggest that the role of the thermal physics through the parameterization of the consistency is significantly minor compared to other parameters such as the basal friction or the power-law index. Therefore, the solving of a high precision model for the temperature field in the bulk could be pointless in the case of a power-law Stokes model and an assumed temperature profile should be sufficient. The present analysis corroborates a large scale numerical experiment performed in [38].

\section{Identification of the scalar-valued triple $(n, m ; \beta)$}

As observed here-before, a major quantity for the control of this type of flow is the friction coefficient $\beta$. The role of the slip ratio appears of great importance on the sensitivities and identification results on both the consistency and the rheological exponent by controlling the flow regime.

The following numerical experiments are performed in the general context of an irregularly perturbed bottom (geophysical like topography), but again, both approaches are applicable for reproducible fluid flows observed during lab experiments.

In the case of ice flow for instance, it is well known that rapid sliding only occurs in the presence of a layer of subglacial sediment called till underneath the ice. Such a layer can for instance be modeled as a non-linear viscous fluid but a classical approach consists in using a non-linear viscous sliding law such as (11) to mimick this behavior.

\subsection{Numerical experiments}

The following numerical experiments aim to identify at the same time the scalar rheological exponent $n$ and the scalar sliding parameters $\beta$ and $m$ (see equation (11)). They are performed on the Mertz glacier flowline domain (see Figure 7) with a non-linear Poiseuille flow prescribed on the left boundary $\Gamma_{l}$ and a lithostatic pressure on the right boundary $\Gamma_{r}$. A non-linear sliding law with $m=3$ (see equation (11)), and a uniform consistency $\eta_{0}$ are considered. In order to explore every sliding situations, three average slip ratios are considered hereafter corresponding to $r=0.01, r=1$ and $r=40$ (see equation (20)). The value $r=40$ corresponds to the maximum slip ratio one can achieve for the present flow with complex topography. As further discussed, the local variation of the slip ratio can be large even for a given scalar friction coefficient $\beta$. Since scalar values are identified the cost function is simply:

$$
j(n, m ; \beta)=\int\left\|u_{s}(n, m ; \beta)-u_{s}^{o b s}\left(n_{t}, m_{t} ; \beta_{t}\right)\right\|_{2}^{2} d \mathbf{x}
$$

The evolution of the cost $j(n, m ; \beta)$ (see (27)) and its gradient with respect to $(n, m ; \beta)$ along the data assimilation cycle is plotted in Figure 17. Figure 17(a) shows a robust identification for every situations; the decreasing of the costs is quite smooth (without taking into account the restarts of the minimization algorithm). The large drop of the gradient at the end highlight the fact that the optimum does not lie in a locally convex "valley" and it requires to run the minimization long enough to achieve the convergence even if the cost seems stable. We point out that such a result requires a sharp minimizer and accurate numerical schemes and 


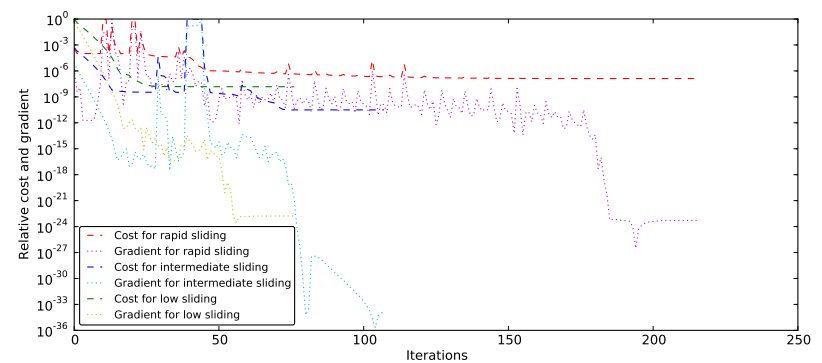

(a) Costs and gradients

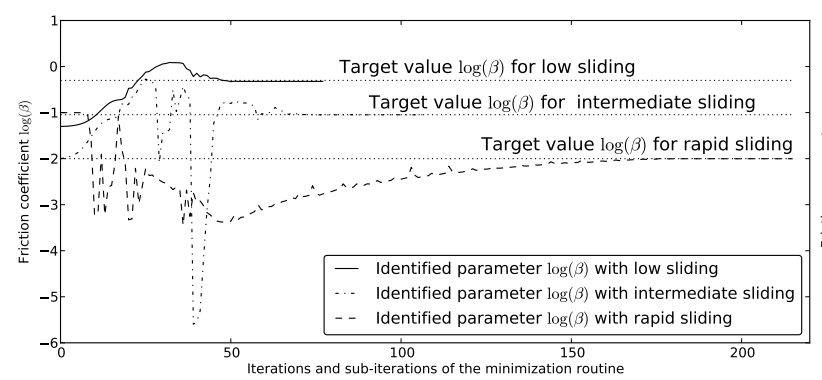

(c) Friction coefficient $\log (\beta)$

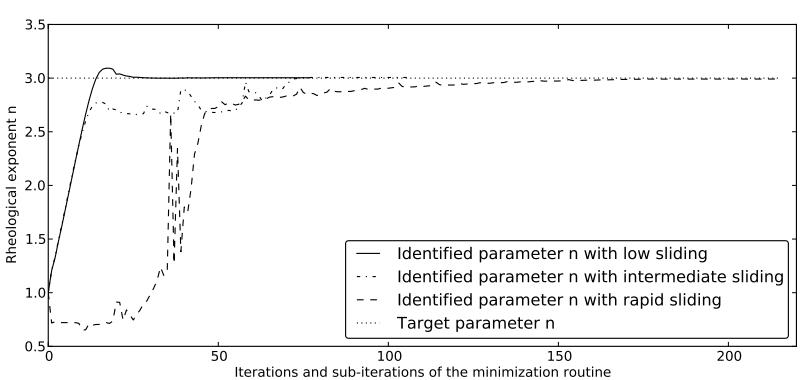

(b) Rheological exponent $n$

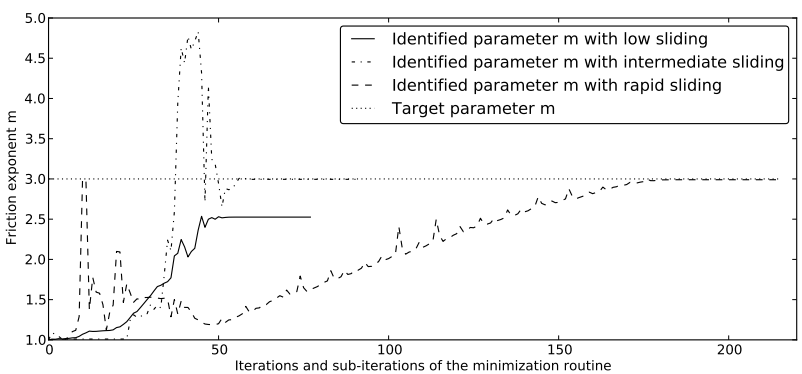

(d) Friction exponent $m$

Figure 17: Identification of the scalar-valued triple $(n, m ; \beta)$.

gradient. We also point out that this behavior is not sensitive to the first guess as long as the initial $n$ is taken smaller than the target one.

\subsection{Discussion}

Inferred values. It clearly appears that the convergence of the rheological exponent $n$ to the target value is achieved before the friction parameters $\beta$ and $m$ start their convergence. It demonstrates that $n$ is a completely dominant parameter in the perspective of controlling the flow. Once $n$ is almost converged, $m$ followed by $\beta$ start to converge to their target values. The errors on the inferred parameters for the different situations are given in Table 2. The final results are fully conclusive; all three parameters are well identified allowing to completely infer the flow regime from horizontal surface velocities only, under the assumption of uniform values for the three parameters. The plot of the resulting slip ratio in the case of low sliding ( $r=0.01$, see Figure 18) allows to understand the reason of a less faithful reconstruction of $\beta$ and $m$. Indeed, a slip ratio $r=0.01$ corresponds to a situation where $1 \%$ of the surface velocities come from the sliding and $99 \%$ come from the viscous deformation. Since a noise of $1 \%$ has been considered on the surface velocity data, it is a priori impossible to achieve an identification of the friction parameters. As we can see, the minimization

\begin{tabular}{|c||c|c|c|}
\hline Slip ratio & $r=0.01$ & $r=1$ & $r=40$ \\
\hline$n$ & $0.111 \%$ & $0.198 \%$ & $0.332 \%$ \\
\hline$\beta$ & $7.18 \%$ & $0.225 \%$ & $0.110 \%$ \\
\hline$m$ & $15.8 \%$ & $0.159 \%$ & $0.332 \%$ \\
\hline
\end{tabular}

Table 2: Relative error for the reconstructed friction coefficient $\beta$, the friction exponent $m$ and the rheological exponent $n$ for different slip ratios.

procedure produced a pair $(\beta, m)$ which provide the same surface velocities as the target one but leading to a higher slip ratio $r=0.016$ to overcome the problem of the noise.

Topography/slip-ratio correlation. The surface velocity data and the resulting surface velocities and basal velocities are plotted in Figure 18,19 and 20 for the three average slip ratios $r=0.01, r=0.1$ and $r=40$ respectively.

The use of a scalar $\beta$ as a friction coefficient can be seen as a simplified modeling approach. However, the non-uniform realistic topography considered here leads to a strongly non-uniform slip ratio with respect to the abscissa. In order to observe their variability of the basal state, the pointwise slip ratios and the opposite of the bedrock variations around its mean slope $\alpha$ (here $\alpha \sim$ $2.10^{-2}$ ) are also plotted in Figures 18, 19 and 20 for the 


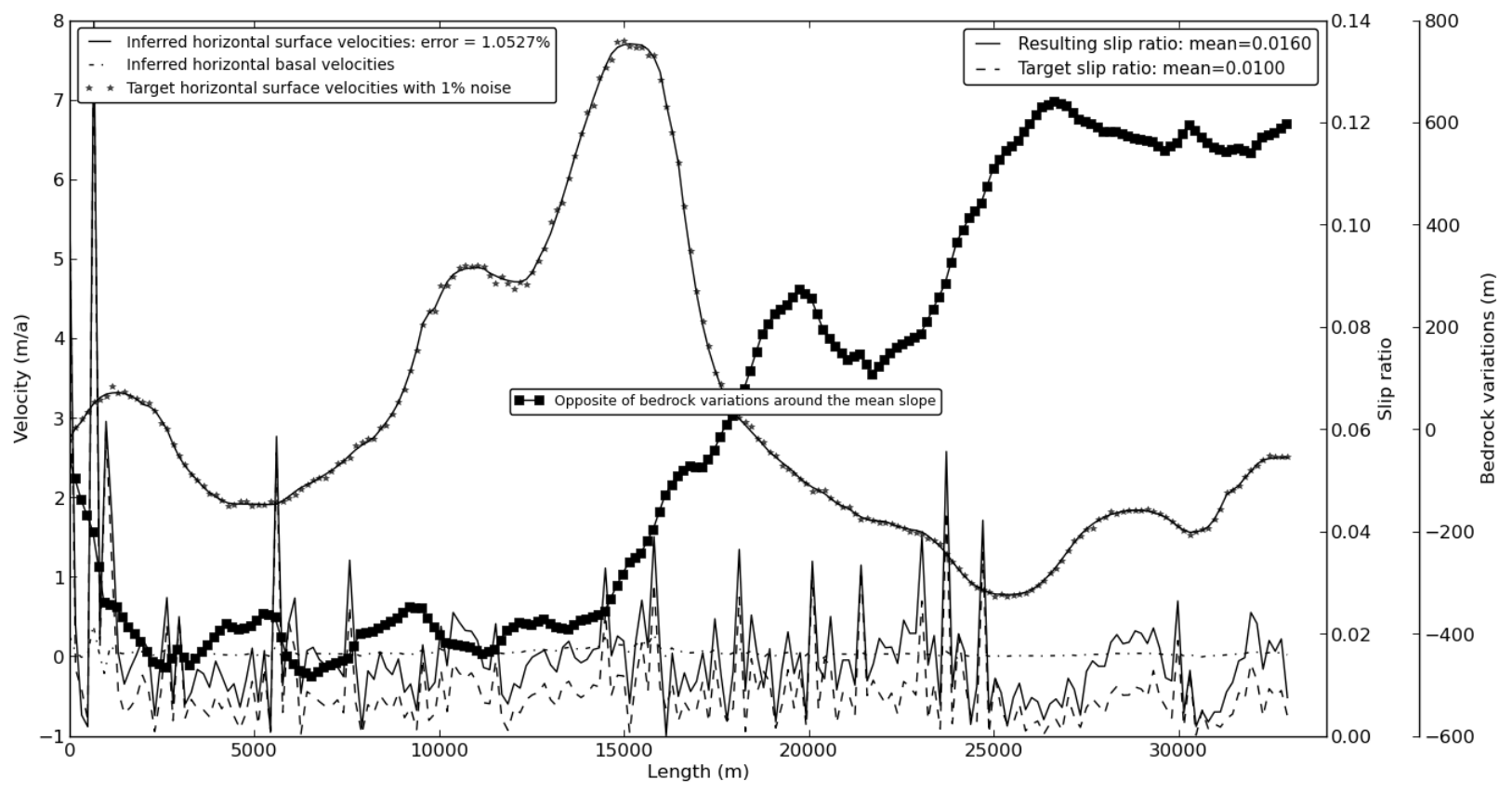

Figure 18: Noisy data and inferred surface velocities, slip ratio and opposite of the bedrock variations for an average slip ratio $r=0.01$

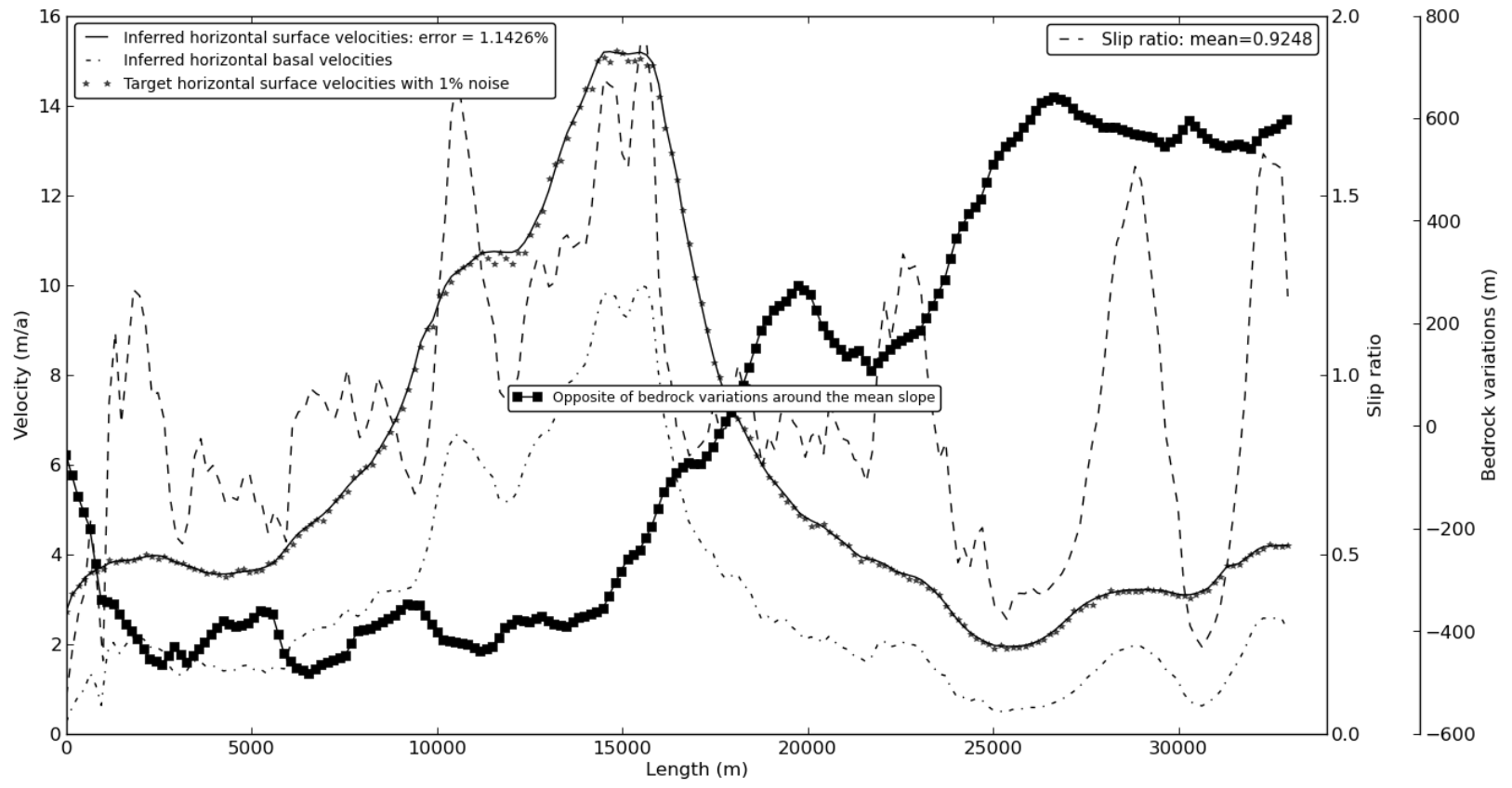

Figure 19: Noisy data and inferred surface velocities, slip ratio and opposite of the bedrock variations for an average slip ratio $r=1$. 


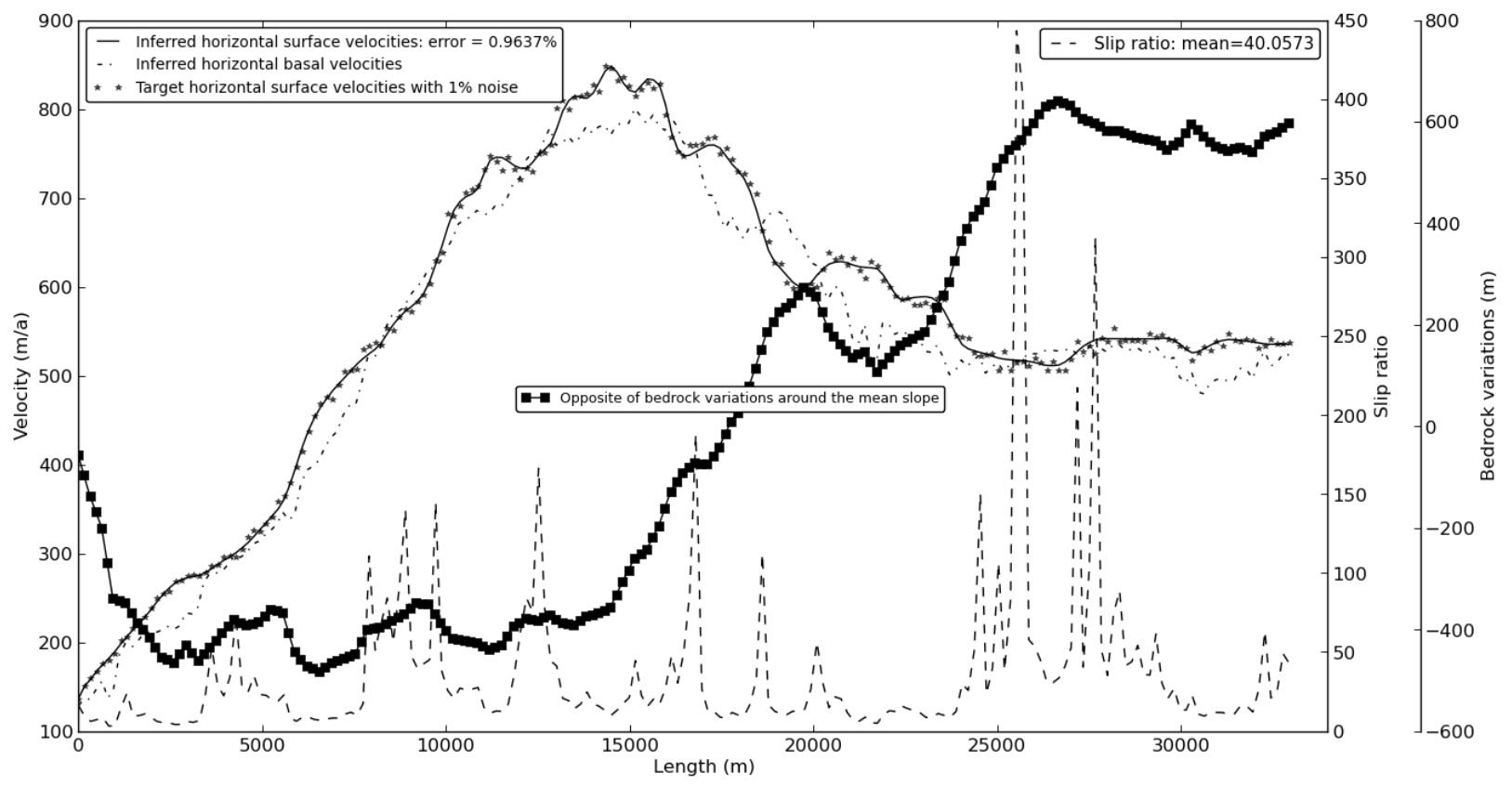

Figure 20: Noisy data and inferred surface velocities, slip ratio and opposite of the bedrock variations for an average slip ratio $r=40$

three situations of sliding.

The case of rapid sliding $(r=40$, the average slip ratio), which allowed to obtain very accurate identification of the three quantities, shows a strongly varying slip ratio; it oscillates between $r=7$ and $r=450$ with large local gradients. A strong correlation between the variations of topography of the bedrock and the slip ratios peaks appears (see Figure 20). This aspect is a major component of the flow since it raises the question of the ability to separate the effect of the friction coefficient from the effect of the topography itself. As a matter of fact, in the ice flow context, the topography of the bedrock is generally the most poorly observed data (compared to the surface velocities and surface topography, see e.g. $[39,15])$.

In the low sliding case $(r=0.01)$, although the correlation between the slip ratio and the topography are not obvious, the slip ratio of the original flow and the one resulting from the identification are very similar in terms of variations.

In conclusion, the identification of the scalar-valued triple $(n, m, ; \beta)$ seems robust. In a more general perspective of infering a spatially varying friction coefficient $\beta(x)$, such an identification allows to strongly constrain the flow and provide an excellent initial guess for a spatial identification of $\beta$. Nevertheless, the observed correlation between the slip-ratio and the topography (mainly in the rapid sliding case $r=40$ ) suggest a potential equifinality on the friction/topography pair.

\section{Equifinality problem of the friction/bed topogra- phy pair}

In the geophysical context, the topography data are generally poorly (or not) observed and the potential correlation with the friction law is of prime interest.

The previous experiment raises a question about the dependency of the inferred friction coefficient on the topography and therefore the ability to separate the two effects. As a final experiment, a spatially varying friction coefficient is identified for a given reference topography and the corresponding reference surface velocity data (with a $1 \%$ Gaussian noise). These reference surface velocity data are then used to perform identifications of the friction coefficient $\beta(x)$ for other topographies of the bedrock. The aim is to assess the link between the two quantities, depending on the regime of sliding, and the ability for the present inverse method to distinguish the two quantities. 


\subsection{Numerical experiment setup}

A $L=50 \mathrm{~km}$ long and $h=1 \mathrm{~km}$ thick domain is considered. The boundary conditions remains the same, as defined in Section 2.1. The reference bedrock $b(x)$ is built as follows:

$$
b(x)=\left\{\begin{array}{l}
a_{0} \sin \left(\frac{2 \pi x}{\omega_{0}}\right)+\alpha_{0}\left(\left(\frac{2 \pi x}{\omega_{1}}\right)+\sin \left(\frac{2 \pi x}{\omega_{2}}\right)\right) \\
\text { if } 0 \leq x \leq 33 \mathrm{~km} \\
a_{1} \sin \left(\frac{2 \pi x}{\omega_{0}}\right)+\alpha_{1}\left(\left(\frac{2 \pi x}{\omega_{1}}\right)+\sin \left(\frac{2 \pi x}{\omega_{2}}\right)\right) \\
\text { if } 33 \mathrm{~km}<x \leq 50 \mathrm{~km}
\end{array}\right.
$$

with $a=\left(a_{0}, a_{1}\right)=(h / 8, h / 16)$ fixed, $\alpha=\left(\alpha_{0}, \alpha_{1}\right)=a / 2 \pm 30 \%$ is randomly generated and $\omega=\left(\omega_{0}, \omega_{1}, \omega_{2}\right)=(50 h, 4 h, 2 h) \pm 30 \%$ is also randomly generated. These values for the variations of the bedrock are chosen according to the bounds on the representable wavelengths assessed in [13]. For the reference domain, $\alpha=(h / 12.7, h / 47.2)$ and $\omega=(51.9 h, 4.4 h, 2.1 h)$. Then, four points $p_{0}, p_{1}$, $p_{2}$ and $p_{3}$ at respectively $x_{0}=0 \mathrm{~km}, x_{1}=16 \mathrm{~km}$, $x_{2}=33 \mathrm{~km}$ and $x_{4}=50 \mathrm{~km}$ are taken on this reference topography, and used as a constraint for the other topographies to be be built; they typically represent measurement points made on the reference (or "true") topography. From there, three topographies are built called the perturbed topography, the large amplitude topography and the piecewise linear topography. The perturbed topography is identical to the reference one with different random vectors $\alpha$ and $\omega$ which are $\alpha=(h / 19.3, h / 42.5)$ and $\omega=(59.3 h, 4.8 h, 1.6 h)$. The large amplitude topography is built on the same principle but replacing $a$ by $\tilde{a}=\left(\tilde{a_{0}}, \tilde{a_{1}}\right)=(h / 4, h / 8)$ and $\alpha$ by $\tilde{\alpha}=\tilde{a} / 2 \pm 30 \%$ randomly generated. The frequencies $\omega$ are randomly perturbed around the same values as before. In the present case, we have $\tilde{\alpha}=(h / 12.3, h / 15.8)$ and $\omega=(58.5 h, 3.63 h, 2.24 h)$. Finally the piecewise linear bottom is just the piecewise linear interpolation of the reference points $p_{0}$, $p_{1}, p_{2}$ and $p_{3}$. The four resulting topographies thus gather at the four reference point and behave according to the formula otherwise. They are plotted in Figure 8.1.

Only four topographies resulting from the randomisation are presented since the results are similar and these four topographies has been chosen as a comprehensive overview of the present discussion.

The surface velocity data are then computed on the reference topography using the following friction coef- ficient:

$$
\beta(x)=\left\{\begin{array}{l}
5.10^{-2} \text { if } 0 \leq x \leq 16 \mathrm{~km} \\
5.10^{-3} \text { if } 16 \mathrm{~km}<x \leq 50 \mathrm{~km}
\end{array}\right.
$$

The combination of friction coefficient and the reference bed topography previously defined leads to define three different areas with three different sliding regimes. The first area for $0 \leq x \leq 16 \mathrm{~km}$ has an average slip ratio $r=2$ and is called the intermediate sliding area. The second area for $16 \mathrm{~km}<x \leq 33 \mathrm{~km}$ has an average slip ratio $r=20$ and is called the rapid sliding area. The last area for $33 \mathrm{~km}<x \leq 50 \mathrm{~km}$ has an average slip ratio $r=200$ and is called the very high sliding area. These areas correspond to the typical sliding regimes observed in large scale Antarctica ice sheds in the coastal regions (analogously to a water shed, see [15]).

The resulting surface velocity data and the corresponding slip ratio along the domain are plotted in Figure 23(a).

We then perform four identifications of the friction coefficient $\beta(x)$ using the same surface velocity data and boundary conditions but on the four different topographies described herebefore. The inferred friction coefficients are plotted in Figure 23(b) and the resulting surface velocities are plotted in Figure 23(a). The cost function used in what follows is defined by:

$j(\beta(x) ; \gamma)=\int_{\Gamma_{s}}\left\|u_{s}^{o b s}-u_{s}(\beta(x))\right\|_{2}^{2} d \mathbf{x}+\gamma \int_{\Gamma_{s}}\left\|\partial_{x} \beta(x)\right\|_{2}^{2} d \mathbf{x}$

The identification of the friction coefficient on the reference bed includes a regularization and the plotted result is optimal in Morozov's sense. Since we will not be able to achieve the Morozov's optimal discrepancy when using the other topographies, the regularization parameter is kept constant and equal to the one adjusted on the optimal case.

Evolutions of the cost function and the gradient along the minimization process for the two different initial guesses are plotted in Figure 22.

\subsection{Discussion}

A first observation is that, in order to obtain very high slip ratio (typically $r>50$ ), it is required to provide an amplitude small enough on the topography (see Figures 


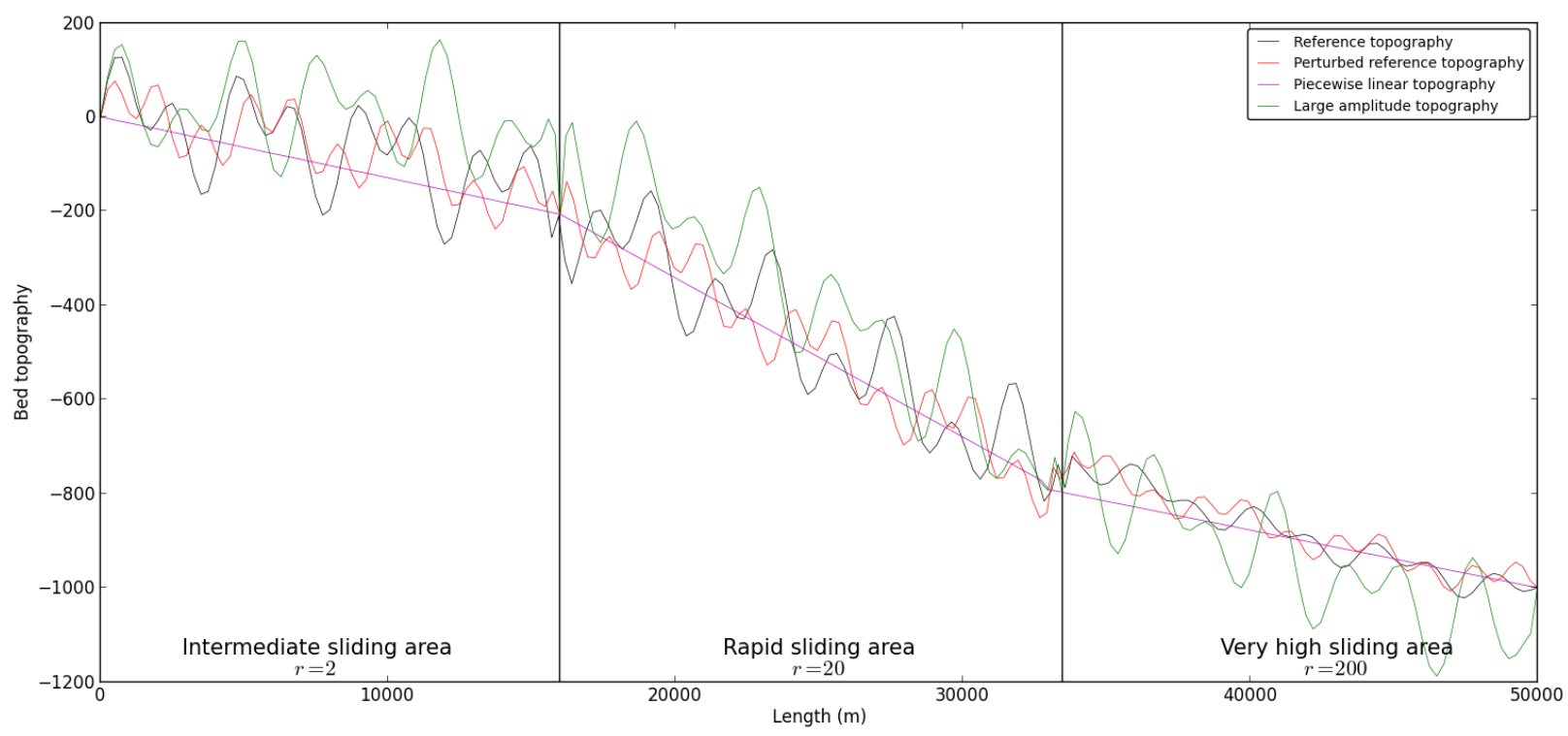

Figure 21: Bed topographies of the four different computational domains as defined in 8.1

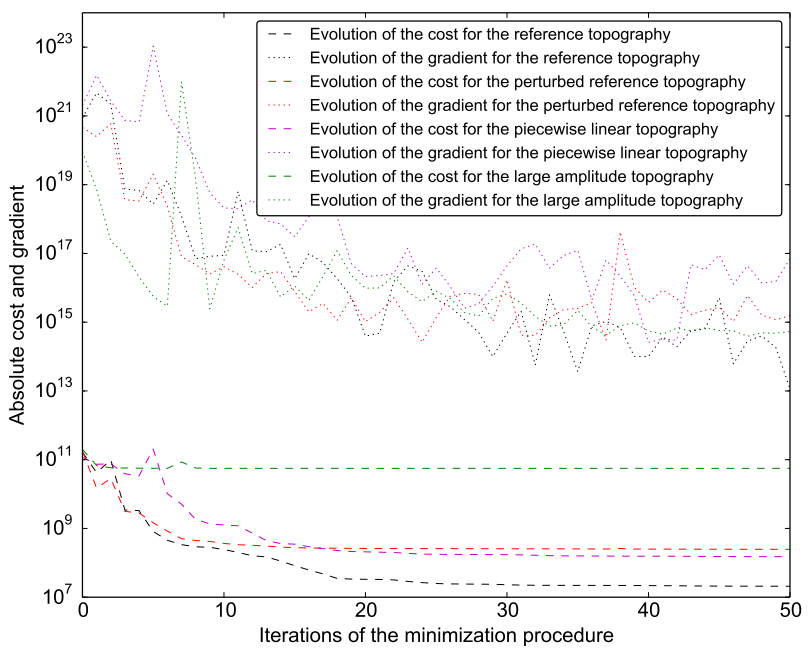

Figure 22: Costs and gradients along the minimization cycle. They are plotted in absolute value in order to compare the results of the different topographies.

8.1 and 8.1). Otherwise, for arbitrarily small $\beta$, the maximum sliding is controlled by the topography peaks. In the present results, the effect is obviously purely bidimensionnal but the global idea also applies on 3D flows.

From the evolution of the cost and the gradient (see Figure 22), we retrieve the fact that the poorest identification is obtained with the large amplitude topography (as observed in the previous paragraph). The perturbed topography and the piecewise linear topography leads to rather similar final cost and obviously the best identification is obtained on the reference topography. Similarly to the identification of the consistency (see Figure 13), the decreasing of the gradient, regardless of the considered topography, is much smaller (4 to 6 orders of magnitude) than for the identification of the rheological exponent $n$ (20 orders of magnitude, see Figure 8$)$ or the identification of the $(\beta, n, m)$ triple (10 to 30 orders of magnitude, see Figure 17(a)) pointing out the ill-posedness of this identification problem.

From Figure 23(a), we observe that the optimal fitting is achieved using the reference bed topography according to Morozov; an $L^{2}$ relative error of $0.9 \%$ is obtained. Figure 23(b) shows a good match between the target friction coefficient and the inferred one. The transition between the two values for the friction coefficient (between $x=9 \mathrm{~km}$ and $x=15 \mathrm{~km}$ ) is relatively smooth due to the regularization term.

The results of the identification using the randomly perturbed topography, yet not optimal according to Morozov, are still very good, providing a relative error between the computed surface velocities and the data of $3.1 \%$. On the contrary, Figure 23(b) shows an inferred friction coefficient very different from the target one for $r \geq 20$, the match in the intermediate sliding area remaining good. 
The same holds for the piecewise linear topography where a very good match of $2.3 \%$ is obtained between computed and target surface velocities, providing a completely different friction coefficient. It is relatively higher in average, due to the removal of any topographical effects. Again, the match in the intermediate sliding area $(r=2)$ is good. Finally on the large amplitude topography, the solution cannot achieve velocities high enough for $r \geq 20$ and the lower threshold imposed on the friction coefficient is obtained everywhere in the area of rapid and very high sliding to provide the highest possible velocities, still far from the observed one. It results in a $50 \%$ relative error between the observed and computed velocities.

These experiments, performed using a highly accurate numerical method (order two finite element schemes, exact adjoint of the full-Stokes problem) hence without any numerical artefact, demonstrate that the inversion of the full-Stokes model with respect to the friction/topography pair is a severely ill-posed problem. It is very likely that this result remains true for $3 \mathrm{D}$ flows. The next question would be to analyze how it affects the predictive capabilities of a calibrated Stokes model for ice-sheets mass balance.

\section{Conclusion}

This work presents, using a second order numerical tool (hence accurate, no numerical artifacts), an adjoint-based inverse approach for inferring the rheology and basal properties of gravity driven free-surface pseudoplastic flows. The present numerical method combines an efficient four-field finite element solver based on an augmented Lagrangian type algorithm for the forward problem and its automatically differentiated counterpart to solve the adjoint problem. Numerical tests assess ratios of four (4) in terms of computational time and memory consumption compared to a classical fixed point approach. In addition, some significant improvements could be made within the element-wise (thus highly-parallelizable) nonlinear iterative part of the algorithm.

A preliminary section addresses the problem of the viscosity singularity at vanishing shear-rate (i.e. at the free-surface) as a limitation for the use of surface velocities to constrain the rheology of the fluid model. Based on sensitivity analyses of a spatially distributed rheological exponent, this section allows to conclude that the convergence to the singularity through refinement of the surface does not affect the forward and adjoint solutions, for different flow regimes (in terms of slip ratio). Therefore, the transmission of the constraints through a stiff surface layer let the surface velocities unchanged and control of the rheological parameters using this type of observations can be done.

The two next sections focus then on the virtual rheometry approach to infer the scalar power-law exponent and the spatially varying consistency using surface velocity observations. The first one shows an extremely robust behavior, allowing to accurately infer the power-law exponent from very noisy data (50\% of noise). Conversely, the identification of the consistency is a severely ill-posed problem and leading to an important equifinality; the role of the consistency in terms of surface velocities is minor and an assumed parameterization to describe this quantity is sufficient. These results are obtained for a wide range of flow regimes, defined by the degree of slip on the base.

These numerical investigations which could be called "virtual rheometry" experiment are valid both for industrial power-law fluids observed in laboratory experiments or geophysical flows (if the negligible inertia assumption is correct).

Next, inspired from the reality of geophysical flows such as glaciers, the two final sections thoroughly investigate the role of the friction coefficient (within a nonlinear viscous sliding law) in conjunction with the rheology and the topography, and the ability for our inverse method to distinguish those non-linearly combined effects.

Recall that the considered geophysical flows features are: uniqueness of the observed flow situation, flow dominated by their basal condition and rheology, observation of the surface of the flow only, poorly observed topography.

We first achieve very accurate joint identifications of the scalar-valued triple $(n, m ; \beta)$ for any degree of slip, providing the ability to completely infer the flow regime solely based on surface velocities. The identification of a scalar valued friction coefficient $\beta$ is however a simplification of the reality but such an identification represent an excellent initial guess towards the identification of a spatially varying friction coefficient. The behavior of the optimization procedure also highlights the need for a highly accurate and robust data assimilation platform (in terms of gradient precision and descent algorithm) for such an identification to be obtained. 
The final section thus provides identifications of the friction coefficient on a flow with various sliding regimes (from intermediate to very high sliding). In order to investigate the joint action of the friction/topography pair, several perturbed bedrock topographies are considered. The results demonstrate a strong equifinality problem through an ability for the inverse model to provide a good match on the surface velocities through very different friction coefficients, depending on the topography, particularly for rapid sliding. Since the observation of the bed topography is generally poor compared to the surface velocities, this result raises an interrogation on the predictive capabilities of a calibrated Stokes model for this type of flow.

\section{Acknowledgments}

This work was partially supported by PRES Toulouse, with the $\mathrm{PhD}$ fund of the first author. It was carried on at the Jet Propulsion Laboratory and the California Institute of Technology under a contract with the National Aeronautics and Space Administration through the President's and Director's Fund Program. The authors want to thank Eric Larour (Jet Propulsion Laboratory) as the project lead for the Ice Sheet System Model (ISSM) and Ronan Madec (Toulouse Institute of Mathematics) for his help on the development of the adjoint model of DassFlow-Ice. The authors also thank Jean-Paul Vila (Mathematics Institute of Toulouse) for fruitful discussions related to the singularity existence at free surface. This work was also supported by Agence Nationale de la Recherche through ADAGe project No. ANR-09-SYSC-001. Finally, the authors want to thank two anonymous reviewers for their helpful and encouraging remarks.

\section{References}

[1] P. Coussot, Rheometry of pastes, suspensions, and granular materials: applications in industry and environment, WileyInterscience, 2005.

[2] C. Ancey, Plasticity and geophysical flows: A review, Journal of Non-Newtonian Fluid Mechanics 142 (1) (2007) 4-35. doi:10.1016/j.jnnfm.2006.05.005.

[3] H. Park, S. Hong, J. Lim, Estimation of rheological parameters using velocity measurements, Chemical Engineering Science 62 (23) (2007) 6806-6815. doi:10.1016/j.ces.2007.08.053.

[4] H. C. Bandulasena, W. Zimmerman, J. Rees, An inverse method for rheometry of power-law fluids, Measurement Science and Technology 22 (12) (2011) 125402. doi:10.1088/0957$0233 / 22 / 12 / 125402$.

[5] S. Nascimento, M. Naccache, F. Rochinha, Identification of non-Newtonian rheological parameter through an inverse formulation, Journal of the Brazilian Society of Mechanical
Sciences and Engineering 32 (2) (2010) 187-194, 00001. doi:10.1590/S1678-58782010000200013.

[6] D. Szeliga, J. Gawad, M. Pietrzyk, Inverse analysis for identification of rheological and friction models in metal forming, Computer Methods in Applied Mechanics and Engineering 195 (48-49) (2006) 6778-6798. doi:10.1016/j.cma.2005.03.015.

[7] J. Glen, The creep of polycrystalline ice, Proceedings of the Royal Society of London. Series A. Mathematical and Physical Sciences 228 (1175) (1955) 519-538. doi:10.1098/rspa.1955.0066.

[8] K. Cuffey, W. S. B. Paterson, The Physics of Glaciers, Academic Press, 2010.

[9] F. Rémy, C. Ritz, L. Brisset, Ice-sheet flow features and rheological parameters derived from precise altimetric topography, Annals of Glaciology 23 (1996) 277-283.

[10] R. W. Griffiths, The dynamics of lava flows, Annual Review of Fluid Mechanics 32 (1) (2000) 477-518. doi:10.1146/annurev.fluid.32.1.477.

[11] R. Champallier, M. Bystricky, L. Arbaret, Experimental investigation of magma rheology at $300 \mathrm{mpa}$ : From pure hydrous melt to 76 vol.\% of crystals, Earth and Planetary Science Letters 267 (3) (2008) 571-583. doi:10.1016/j.epsl.2007.11.065.

[12] N. Petra, H. Zhu, G. Stadler, T. Hughes, O. Ghattas, An inexact Gauss-Newton method for inversion of basal sliding and rheology parameters in a nonlinear Stokes ice sheet model, Journal of Glaciology 58 (2012) 889-903. doi:10.3189/2012JoG11J182.

[13] N. Martin, J. Monnier, Adjoint accuracy for the full Stokes ice flow model: limits to the transmission of basal friction variability to the surface, The Cryosphere 8 (2) (2014) 721-741. doi:10.5194/tc-8-721-2014

[14] N. Martin, J. Monnier, Four-field finite element solver and sensitivities for quasi-Newtonian flows, Accepted in SIAM - Journal of Scientific Computing.

[15] E. Rignot, J. Mouginot, B. Scheuchl, Ice flow of the Antarctic ice sheet, Science 333 (6048) (2011) 1427-1430. doi: $10.1126 /$ science. 1208336.

[16] P. Noble, J.-P. Vila, Thin power-law film flow down an inclined plane: consistent shallow-water models and stability under large-scale perturbations, Journal of Fluid Mechanics 735 (2013) 29-60.

[17] R. Johnson, R. McMeeking, Near-surface flow in glaciers obeying Glen's law, The Quarterly Journal of Mechanics and Applied Mathematics 37 (2) (1984) 273-291.

[18] G. Astarita, G. Marrucci, Principles of non-Newtonian fluid mechanics, McGraw-Hill Book Company, 1974.

[19] M. Boutounet, J. Monnier, J.-P. Vila, Multi-regime shallow freesurface flow models for power-law fluids, submitted.

[20] A. C. Fowler, Mathematical geoscience, Vol. 36, Springer, 2011.

[21] D. Sandri, A posteriori estimators for mixed finite element approximations of a fluid obeying the power law, Computer Methods in Applied Mechanics and Engineering 166 (3) (1998) 329_ 340. doi:10.1016/S0045-7825(98)00094-2.

[22] J.-C. Gilbert, C. Lemaréchal, Some numerical experiments with variable-storage quasi-newton algorithms, Mathematical Programming 45 (1-3) (1989) 407-435. doi:10.1007/BF01589113.

[23] M. Honnorat, J. Marin, J. Monnier, X. Lai, Dassflow v1.0: a variational data assimilation software for $2 \mathrm{D}$ river flows. URL http: //hal .inria.fr/inria-00137447

[24] Dassflow: Data assimilation for free surface flows http:// ww-gmm.insa-toulouse.fr/ monnier/DassFlow.

[25] J. Monnier, in: Variational data assimilation, From optimal control to large scale data assimilation, Open Learn. Res., Courses University of Toulouse, 2013, http://pedagotech. inp-toulouse.fr/130107. 
[26] N. Martin, Modélisation directe et inverse d'écoulements géophysiques viscoplastiques par méthodes variationnelles : Application à la glaciologie, Ph.D. thesis, supervised by Monnier, Jérôme, INSA de Toulouse (2013).

[27] Dassice: Data assimilation for ice flows https://sites. google.com/site/webpageofnathanmartin/dassice.

[28] A. Griewank, et al., On automatic differentiation, Mathematical Programming: recent developments and applications 6 (1989) 83-107.

[29] P. R. Amestoy, I. S. Duff, J.-Y. L'Excellent, J. Koster, A fully asynchronous multifrontal solver using distributed dynamic scheduling, SIAM Journal on Matrix Analysis and Applications 23 (1) (2001) 15-41. doi:10.1137/S0895479899358194.

[30] L. Hascoët, V. Pascual, Tapenade 2.1 user's guide. URL http://hal .inria.fr/inria-00069880

[31] H. Lee, Optimal control for quasi-Newtonian flows with defective boundary conditions, Computer Methods in Applied Mechanics and Engineering 200 (33) (2011) 2498-2506. doi:10.1016/j.cma.2011.04.019.

[32] G. Chambon, A. Ghemmour, D. Laigle, Gravity-driven surges of a viscoplastic fluid: An experimental study, Journal of Non-Newtonian Fluid Mechanics 158 (1) (2009) 54-62. doi:10.1016/j.jnnfm.2008.08.006.

[33] K. Hutter, Dynamics of glaciers and large ice masses, Annual Review of Fluid Mechanics 14 (1) (1982) 87-130.

[34] J. S. Greenbaum, D. D. Blankenship, D. A. Young, T. G. Richter, B. Legresy, B. Galton-Fenzi, Y. Gim, Basal characteristics and inferred bathymetry beneath the Mertz glacier tongue, Antarctica from coupled airborne radar sounding and gravity prior to the february 12th 2010 breakup event, in: 4th SCAR Open Science Conference - Antarctica: Witness to the Past and Guide to the Future. Submitted Abstracts, 2010.

[35] M. Filippucci, A. Tallarico, M. Dragoni, A three-dimensional dynamical model for channeled lava flow with nonlinear rheology, Journal of Geophysical Research 115 (B5) (2010) B05202. doi:10.1029/2009JB006335.

[36] P. Schenk, R. Wilson, A. Davies, Shield volcano topography and the rheology of lava flows on Io, Icarus 169 (1) (2004) 98-110. doi:10.1016/j.icarus.2004.01.015.

[37] C. Vogel, Computational Methods for Inverse Problems, SIAM, 2002.

[38] H. Seroussi, M. Morlighem, E. Rignot, A. Khazendar, E. Larour, J. Mouginot, Dependence of century-scale projections of the Greenland ice sheet on its thermal regime, Journal of Glaciology 59 (218) (2013-12-01T00:00:00) 1024-1034. doi:doi:10.3189/2013JoG13J054.

[39] P. Fretwell, H. Pritchard, D. Vaughan, J. Bamber, N. Barrand, R. Bell, C. Bianchi, R. Bingham, D. Blankenship, G. Casassa, et al., Bedmap2: improved ice bed, surface and thickness datasets for Antarctica, The Cryosphere 7 (1) (2013) 375-393. doi:10.5194/tc-7-375-2013. 


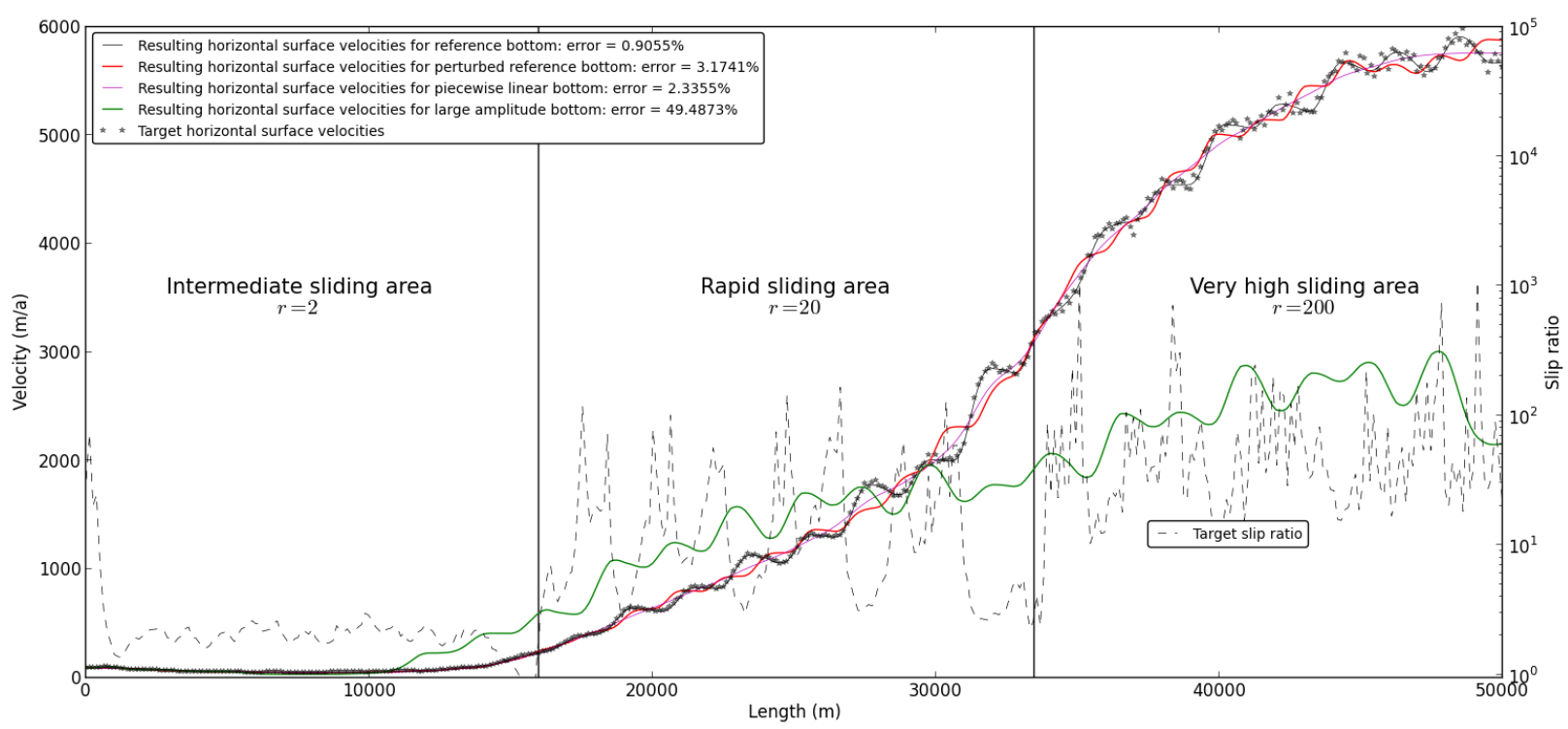

(a) Target surface velocities and surface velocities resulting from the minization

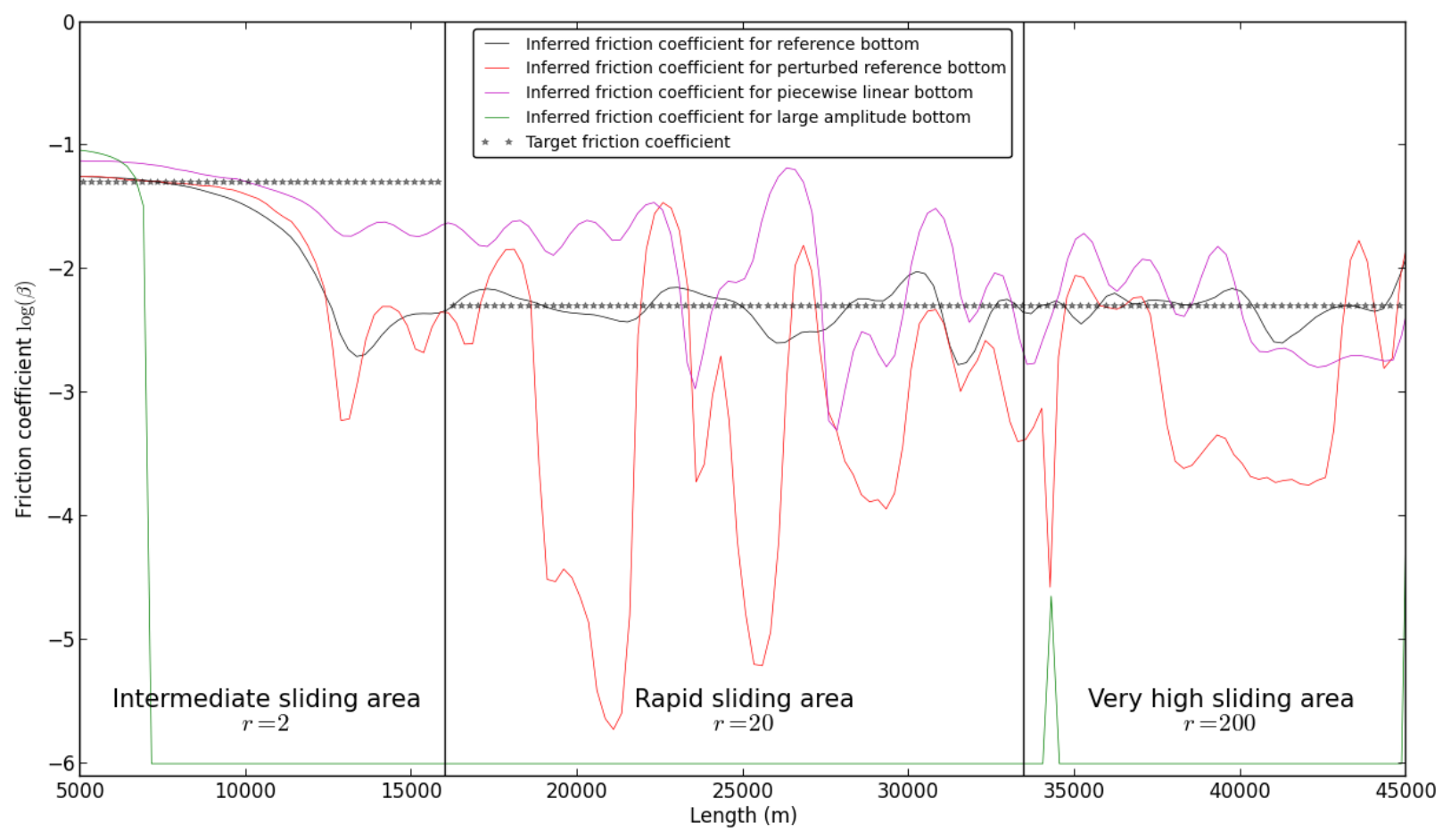

(b) Target and inferred friction coefficient 